\title{
On the choice of parameters in Singular Spectrum Analysis and related subspace-based methods
}

\author{
Nina Golyandina
}

In the present paper we investigate methods related to both the Singular Spectrum Analysis (SSA) and subspacebased methods in signal processing. We describe common and specific features of these methods and consider different kinds of problems solved by them such as signal reconstruction, forecasting and parameter estimation. General recommendations on the choice of parameters to obtain minimal errors are provided. We demonstrate that the optimal choice depends on the particular problem. For the basic model 'signal + residual' we show that the error behavior depends on the type of residuals, deterministic or stochastic, and whether the noise is white or red. The structure of errors and the convergence rate are also discussed. The analysis is based on known theoretical results and extensive computer simulations.

AMS 2000 Subject Classifications: Primary 62M20, 62F10, 62F12; secondary 60G35, 65C20, 62G05.

Keywords And Phrases: Singular Spectrum Analysis, Time series analysis, Subspace-based methods, Signal processing, Forecasting, Linear recurrent formula, ESPRIT, Frequency estimation.

\section{INTRODUCTION}

In the 1970-80s many papers describing methods based on the Singular Value Decomposition (SVD) of a specially constructed Hankel matrix have been published, see $[4,6,7,11,22,25]$ among many others. Many modern methods are based on the ideas proposed in these papers and these methods have proved to be very useful in many applied areas. The list of the methods includes HSVD (Hankel SVD, [3]) and HTLS (Hankel Total Least Squares, [35]) which are mostly used in the nuclear magnetic resonance spectroscopy, ESPRIT [30] used in the Direction-Of-Arrival problems, and Singular Spectrum Analysis (SSA) which has been applied in many different areas.

All these methods have several common features (up to using the same algorithms), but each of them also has some special features. The purpose of this paper is to show some commonalities and specifics of these methods and to analyze the optimal choice of parameters. Since SSA is not limited

arXiv: 1005.4374 to any particular area of application, we mostly base our investigation on SSA.

Let us briefly describe Basic SSA [15] for the real-valued case. Let us observe a one-dimensional time series $F_{N}=$ $\left(f_{0}, \ldots, f_{N-1}\right)$ of length $N$. We suppose that $F_{N}$ is a sum of several unknown but identifiable components and we are interested in some of them, for example, trend or regular oscillations.

Embedding Given a window length $L(1<L<N)$ one proceeds with constructing $K=N-L+1$ lagged ( $L$-lagged) vectors $X_{i}=\left(f_{i-1}, \ldots, f_{i+L-2}\right)^{\mathrm{T}}, 1 \leq i \leq K$, and composing them into the matrix $\mathbf{X}=\left[X_{1}: \ldots: X_{K}\right]$, which is called the $L$-trajectory matrix. Note that $\mathbf{X}$ is a Hankel matrix.

Decomposition The key step in SSA is the SVD of the trajectory matrix:

$$
\mathbf{X}=\sum_{i=1}^{d} \sqrt{\lambda_{i}} U_{i} V_{i}^{\mathrm{T}},
$$

where $\lambda_{1} \geq \cdots \geq \lambda_{d}>0$ are the positive eigenvalues of the matrix $\mathbf{C}=\mathbf{X} \mathbf{X}^{\mathrm{T}}$ arranged in the nonincreasing order, $\left\{U_{1}, \ldots, U_{d}\right\}$ is the orthonormal system of the corresponding eigenvectors of the matrix $\mathbf{C}\left(U_{i}\right.$ are left singular vectors of $\mathbf{X})$, and $V_{i}=\mathbf{X}^{\mathrm{T}} U_{i} / \sqrt{\lambda_{i}}, i=1, \ldots, d$, are the factor vectors (right singular vectors). Note that $U_{1}, \ldots, U_{d}$ form an orthonormal basis of the column space $\mathcal{L}_{d}$ of $\mathbf{X}$, which is called the trajectory space, $\mathcal{L}_{d}=\operatorname{span}\left(U_{1}, \ldots, U_{d}\right)$. The triple $\left(\sqrt{\lambda_{i}}, U_{i}, V_{i}\right)$ is called the $i$-th eigentriple (or ET) and $\sqrt{\lambda_{i}}$ is called the $i$-th singular value of $\mathbf{X}$.

Grouping After grouping the eigentriples by choosing a partition of the set $\{1, \ldots, d\}$ onto $m$ disjoint subsets $I_{1}, \ldots, I_{m}$, one obtains the decomposition

$$
\mathbf{X}=\mathbf{X}_{I_{1}}+\cdots+\mathbf{X}_{I_{m}},
$$

where $\mathbf{X}_{I}=\sum_{i \in I} \sqrt{\lambda_{i}} U_{i} V_{i}^{\mathrm{T}}$. Alternatively, we can consider the grouping step as a decomposition of the trajectory space into the orthogonal sum of subspaces: $\mathcal{L}_{d}=\bigoplus_{k=1}^{m} \mathcal{L}^{(k)}$, where $\mathcal{L}^{(k)}=\operatorname{span}\left(U_{i}, i \in I_{k}\right)$.

Reconstruction (diagonal averaging) One can reconstruct the components of the original series by the diagonal 
averaging of each component $\mathbf{X}_{I_{k}}$ :

$$
F_{N}=\widetilde{F}^{(1)}+\cdots+\widetilde{F}^{(m)},
$$

where $\widetilde{F}^{(k)}=\left(\widetilde{f}_{0}^{(k)}, \ldots, \widetilde{f}_{N-1}^{(k)}\right)$ and $\widetilde{f}_{i}^{(k)}$ is the average value along the $i$-th secondary diagonal of $\mathbf{X}_{I_{k}}$.

Although the described algorithm is designed to decompose the original time series into a sum of an arbitrary number of components, we will be considering the problem of decomposition into a sum of two time series (shortly, t.s.) components. The problem of decomposition into several components can be reduced to the sequential extraction of the components one by one (e.g., first by extracting the trend with a small window length and then by extracting the periodicity from the residual using a large window length, see [15]). In addition, a considerable number of methods of time series analysis (including ESPRIT examined in this paper) solve the problem of analyzing noisy signals and, in fact, consider only two t.s. components: signal and noise.

Thus, suppose that we observe $F_{N}=S_{N}+R_{N}$, where $S_{N}$ is the component of interest (e.g., signal) and $R_{N}$ is the residual component. The residual $R_{N}$ can be either a random noise or a deterministic component, or a mixture of both.

The estimator $\widetilde{S}_{N}=\widetilde{F}^{(1)}$ of the time series $S_{N}$ (so called reconstructed signal) can be considered as the main result of application of SSA. Appropriate reconstruction of the signal can be obtained in the case of its approximate separability from the residual. Typical examples of pairs of approximately separable t.s. components (see [15] for details) are trend (i.e., a slowly-varying component) and noise, trend and cyclic components, and two cyclic components with different frequencies. In general, we can talk about approximate separability of one of the following t.s. components: trend, cycle or noise, from a mix of the other ones.

Suppose that the t.s. component $S_{N}$, which we are interested in, satisfies the following two properties. First, let $S_{N}$ be deterministic. Second, let $S_{N}$ be leading, i.e. $S_{N}$ generates the $r$ leading SVD components in the decomposition (1) with indices from $I=\{1, \ldots, r\}$. In fact, we suppose that the minimal singular value of the trajectory matrix $\mathbf{S}$ of $S_{N}$ is larger or asymptotically larger than the maximal singular value of the trajectory matrix $\mathbf{R}$ of $R_{N}$.

The deterministic structure of $S_{N}$ and its approximate separability imply that $S_{N}$ can be approximated by a time series of finite $L$-rank [15]. This means that in (1) all except the $r$ leading eigenvalues are close to zero. In this paper we investigate the asymptotic properties as the time series length $N$ tends to infinity. We consider an infinite time series $F=\left(f_{0}, \ldots, f_{n}, \ldots\right)$ and analyze the finite-length time series $F_{N}$ consisting of its first $N$ terms. For an infinite time series $S$, the following assertion is valid: $S$ has finite rank $r$ (its $L$-trajectory space $\mathcal{L}_{r}^{(\mathrm{s})}$ has dimension $r$ for any $L \geq r$ ) iff $S$ is governed by a linear recurrent formula (LRF) [15]. For finite-rank subseries $S_{N}$ the SVD of $L$-trajectory matrix $\mathbf{S}$ has exactly $r$ positive singular values and $\mathbf{S}$ is rank deficient. If $S_{N}$ is interpreted as a signal, then its trajectory space $\mathcal{L}_{r}^{(\mathrm{s})}$ is called the signal subspace.

Note that if a time series is governed by an LRF, then it can be represented as a sum of products of polynomials, exponentials and sinusoids, and in this case it makes sense to use a parametric setup for the problem. On the other hand, the SSA algorithm of signal reconstruction is essentially nonparametric and can be applied to a class of time series which is wider than the set of perturbed finite-rank signals.

Statements of problems within the framework of SSA can differ in the following aspects.

A1 Features of interest: we can be interested either in the signal $S_{N}$ as a whole or in some of its characteristics. In particular, if $S_{N}$ has finite rank, then it has a known parametric form and we can be interested in parameter estimation. The most elaborated problem is the estimation of damping factors and frequencies of exponentially damped sinusoids in noise. For solving this estimation problem it is sufficient to know only $r$ leading eigenvectors in (1), more specifically, the subspace spanned by these eigenvectors (i.e., the estimated subspace of the signal $S_{N}$ ); see e.g. ESPRIT-like methods.

A2 Type of residuals: the residual $R_{N}$ is either deterministic or stochastic (or it has both random and deterministic components). These cases correspond to different properties of the SSA decomposition and cause different characteristics of estimators. For example, a finite-rank $S_{N}$ will be the leading component if the deterministic residual is bounded by some constant, while white noise can have any variance for large time series lengths $N$ and window lengths $L$. Also, the structure of the stochastic noise (e.g., white or red) can influence the behavior of estimation errors.

A3 Choice of the window length: either we can vary the window length $L$ or $L$ is fixed. In the former case, the problem of the optimal choice of $L$ arises. Then, the asymptotic behavior depends on whether $L$ tends to infinity as $N \rightarrow \infty$ or not. If we consider a matrix $L \times K$ with a fixed number of rows as an input, then the only way is to fix $L$ and to consider $K$ tending to infinity. The other possible reason for choosing a not very large $L$ is the computational cost. However, there are recent computational advances, which make calculations very fast, see [20].

In this paper, we consider different statements of the problem within Aspect A1 and then analyze errors and parameter choice rules following Aspects A2 and A3.

The main information about the time series structure that we obtain within the framework of SSA is contained in the set of eigentriples $\left(\sqrt{\lambda_{i}}, U_{i}, V_{i}\right)$. Consequently we obtain not only the reconstructed signal but also much additional 
information about $S_{N}$. In addition to the problem of reconstructing $S_{N}$, we consider the problems of signal forecasting and signal parameter estimation.

Note that the SVD is determined only by the set of eigenvectors $U_{i}$, since $\lambda_{i}=\left\|\mathbf{X}^{\mathrm{T}} U_{i}\right\|^{2}$ and $V_{i}=\mathbf{X}^{\mathrm{T}} U_{i} / \sqrt{\lambda_{i}}$, where $\mathbf{X}$ is the trajectory matrix of the observed time series. Consequently, $\widetilde{\mathbf{S}}=\sum_{i=1}^{r} U_{i} U_{i}^{\mathrm{T}} \mathbf{X}=\mathrm{P}_{r} \mathbf{X}$, where $\mathrm{P}_{r}$ is the orthogonal projection on $\mathcal{L}_{r}$. Therefore, we can say that the set of eigenvectors (along with the time series $F_{N}$ ) completely determines the whole SVD expansion and, therefore, the results of forecasting and parameter estimation. Thus it is natural to start the investigation with the estimation of eigenvectors or, equivalently, the eigenspace estimation. Note that the problem of estimation of the factor vectors $V_{i}$ becomes the problem of estimation of the eigenvectors $U_{i}$ by changing the window length from $L$ to $N-L+1$.

Let us remark that the transformations applied to the eigenvectors in order to obtain, for example, the frequency estimates can tremendously change the structure of estimation errors. Nevertheless, in Section 2 we consider the errors of signal subspace estimation as the starting point of the investigation. Sections 3 and 4 contain the results on reconstruction and forecasting based on the chosen subspace. Section 5 is devoted to parameter estimation within the framework of the subspace-based methods of signal processing including ESPRIT. In Sections 2-5 we give general recommendations on the choice of the window length $L$ which are based on simulations and known theoretical results. In Section 6 we consider the convergence rate in different conditions (a fixed window length $L$ or a window length proportional to the time series length) for the problems investigated in previous sections.

Let us remark that the results of Sections 2-6 are valid under the condition of strong separability of the signal from the residual. Section 7 deals with several examples, in which there is no strong separability. In Section 8 we consider several versions of Basic SSA and demonstrate some examples of application of the versions designed for the stationary time series to non-stationary ones.

In Section 9 we briefly describe some origins of the SVD providing the key step of the SSA algorithm. We are interested in these origins, since they imply different views on the problem statement and on the parameter choice.

\section{SIGNAL SUBSPACE}

We will generally rely on the results of the paper [27], which is devoted to the discussion of convergence and also contains the main error terms and their upper bounds.

As a measure of the error for the subspace approximation we consider the spectral norm of the difference of projectors on the true subspace and the estimated subspace. Note that this norm is equal to the sine of the largest principal angle between these subspaces. The aim of this section is to investigate the dependence of the approximation error on the window length.
Let $S_{N}$ be a signal of rank $r$. By $\mathrm{P}_{r}^{(\mathrm{s})}$ we denote the orthogonal projector on the signal subspace $\mathcal{L}_{r}^{(\mathrm{s})}$, which is spanned by the left singular vectors $U_{1}^{(\mathrm{s})}, \ldots, U_{r}^{(\mathrm{s})}$ of the signal trajectory matrix $\mathbf{S}$, and by $\mathrm{P}_{r}$ we denote the orthogonal projector on the estimated signal subspace $\mathcal{L}_{r}=$ $\operatorname{span}\left(U_{1}, \ldots, U_{r}\right)$, where $U_{1}, \ldots, U_{r}$ are the $r$ leading left singular vectors of the trajectory matrix of the observed time series $F_{N}$. Note that we can easily calculate the estimation error $\left\|\mathrm{P}_{r}^{(\mathrm{s})}-\mathrm{P}_{r}\right\|$, since the cosine of the largest principal angle between $\mathcal{L}_{r}^{(\mathrm{s})}$ and $\mathcal{L}_{r}$ is equal to the $r$-th eigenvalue of the matrix $\mathbf{U}_{r}^{(\mathrm{s})} \mathbf{U}_{r}^{\mathrm{T}}$, where $\mathbf{U}_{r}^{(\mathrm{s})}=\left[U_{1}^{(\mathrm{s})}: \ldots: U_{r}^{(\mathrm{s})}\right]$ and $\mathbf{U}_{r}=\left[U_{1}: \ldots: U_{r}\right]$ (see e.g. $[5$, p. 18$]$ ).

Let us consider five examples of time series $f_{n}=s_{n}+r_{n}$, $n=1, \ldots, N$,

$$
\begin{array}{ll}
s_{n}=1, \quad r_{n}=-c(-1)^{n}, \\
s_{n}=b^{n} \cos (2 \pi n / 10), & r_{n}=c \\
s_{n}=b^{n} \cos (2 \pi n / 10), & r_{n}=\sigma \varepsilon_{n}, \\
s_{n}=b^{n} \cos (2 \pi n / 10), & r_{n}=\left(\sigma \varepsilon_{n}+c\right) / \sqrt{2}, \\
s_{n}=b^{n} \cos (2 \pi n / 10), & r_{n}=\sigma \eta_{n} .
\end{array}
$$

Here $\varepsilon_{n}$ is a white gaussian noise with variance 1 and $\eta_{n}$ is the autoregressive process of order 1 (red noise) with parameter $\alpha$ and variance $\mathbf{D} \eta_{n}=1$, that is, $\eta_{n}=\alpha \eta_{n-1}+\epsilon_{n}$, where $\epsilon_{n}$ has variance $1-\alpha^{2}$. In this section, we set $c=\sigma=0.1$, $\alpha=0.5, b=1$, and $N=100$.

We choose the level of noise in the time series (3)-(6) to have the same signal-to-noise ratio (SNR), which is conventionally determined as the ratio of the average of squared signal values to the average of squared residual values (or to the variance of residuals if they are random).

Generally, the SNR does not determine the size of the errors of estimates obtained by the SSA processing, since the SNR does not take into consideration the time series length. In fact, the SNR can be used to compare the quality of processing of time series of equal lengths. However, we cannot say that SSA separates signal and noise only if the SNR is larger than a specific value; for example, for any small SNR a sine-wave signal is asymptotically separated from a white noise as $N \rightarrow \infty$ and $L \sim \beta N, 0<\beta<1$.

The time series (2) is included, since we can compare the results with those in [27, Section 4.2.1]. It appears that the main term of perturbation found in [27] is almost equal to the whole error. Moreover, the behavior of errors depends on whether the lengths of the window and the time series are even or odd. In addition, this is an example of a time series that produces the projector error having the first-order term with respect to the perturbation level which is not the main term of the error as $N \rightarrow \infty$.

The time series (3)-(5) differ in the structure of residuals: deterministic, random, or combined. The time series (6) is used to consider a noise that differs from the white noise.

In the case of random residuals, we compute either MSD (mean square deviation) or RMSE (square root of mean 


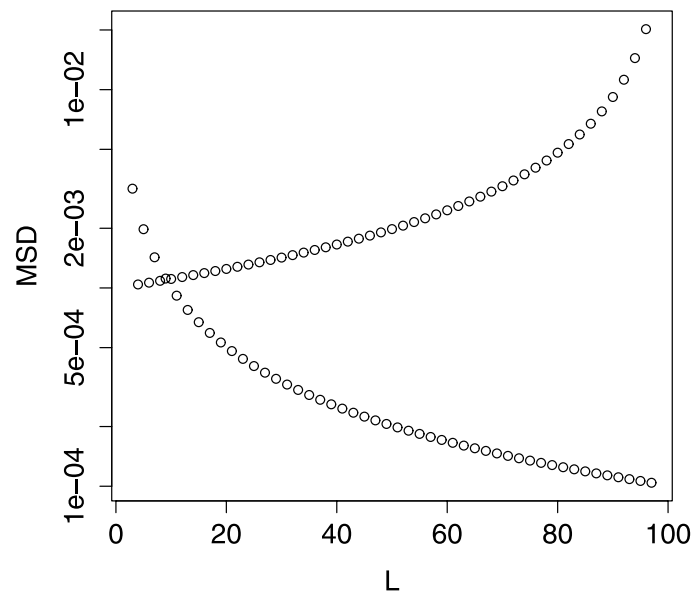

Figure 1. MSD of projector estimates: deterministic residuals, t.s. (2) (log-scale).

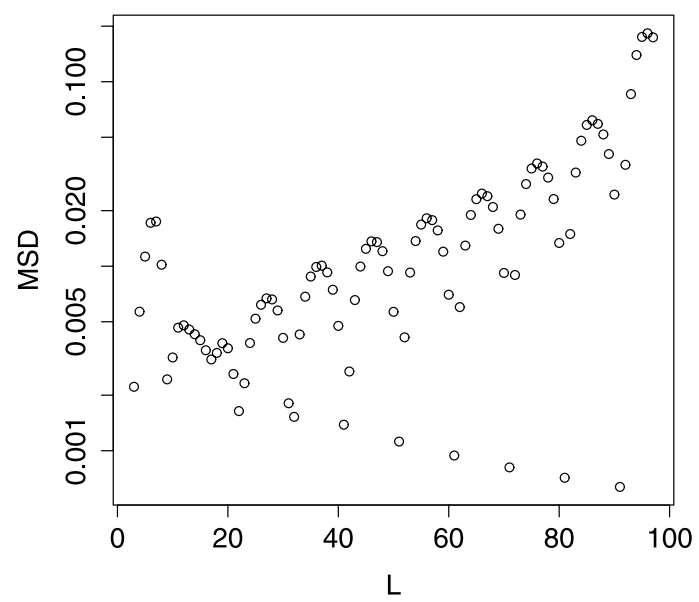

Figure 2. MSD of projector estimates: deterministic residuals, t.s. (3) (log-scale).

square error) as a measure of accuracy. Generally, these criteria yield very similar results. The difference between them is that we can compute the square root before averaging (MSD) or after averaging (RMSE) for the simulation results. In the examples of this section we estimate MSD using 100 simulations.

We present the results of simulation study for the time series (2)-(6) in Figures 1-7. Figures 1 and 2 show the errors in estimation of the projector $\mathrm{P}_{r}^{(\mathrm{s})}$ on the signal subspace for the examples with deterministic residuals. One can see a tendency for the errors to increase as the window length increases. However, for window lengths $L$ that are divisible by periods of the time series components, errors generally decrease. This reflects the influence of the multiplicity of $L$ and/or $K$ to the periods of time series components. Note that if both $L$ and $K$ are divisible by the periods (by 2 in

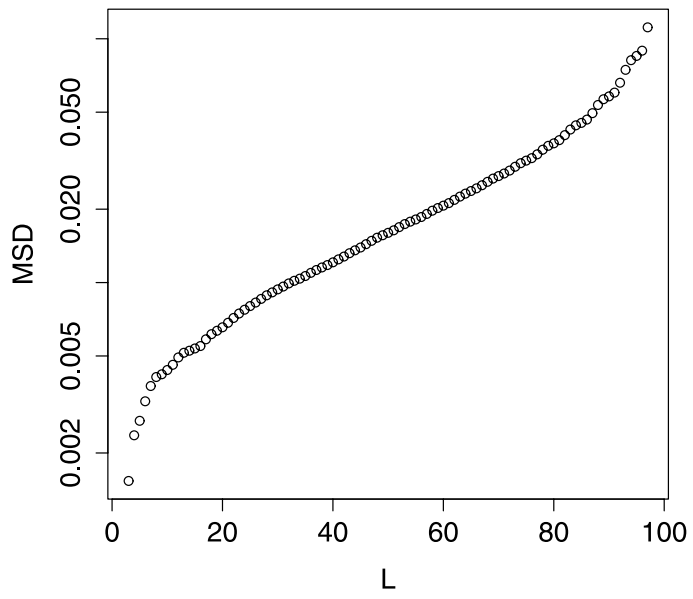

Figure 3. MSD of projector estimates: white-noise residuals, t.s. (4) (log-scale).

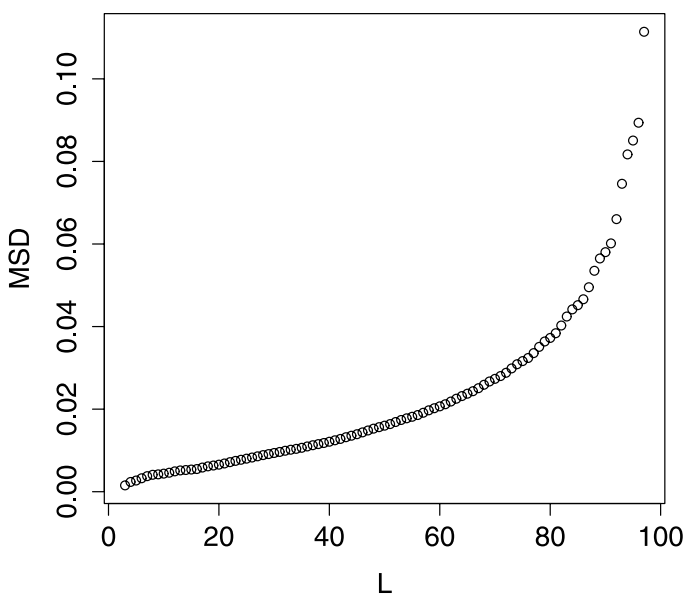

Figure 4. MSD of projector estimates: white-noise residuals, t.s. (4) (original scale).

the first example and by 10 in the second one), then the projector perturbation is equal to 0 . This corresponds to the case of bi-orthogonality of the trajectory matrices of $S_{N}$ and $R_{N}$ and therefore to the case of exact separability. If only $L$ (or $K$ ) is divisible by the period, then this case can be called left (or right) orthogonality. Thus, if the residual is deterministic and $S_{N}$ contains a periodic component, then we observe two effects: the specific behavior of errors in the case of window lengths divisible by the period and a periodic behavior of errors in the general case.

It is clear that if the residual $R_{N}$ contains noise, then the exact orthogonality cannot be achieved. Figures 3 (the logarithmic scale) and 4 (the original scale) demonstrate that the decrease of errors for special window lengths does not occur.

In the case of combined residuals (Fig. 5), the behavior of projector errors inherits the properties of errors for both 


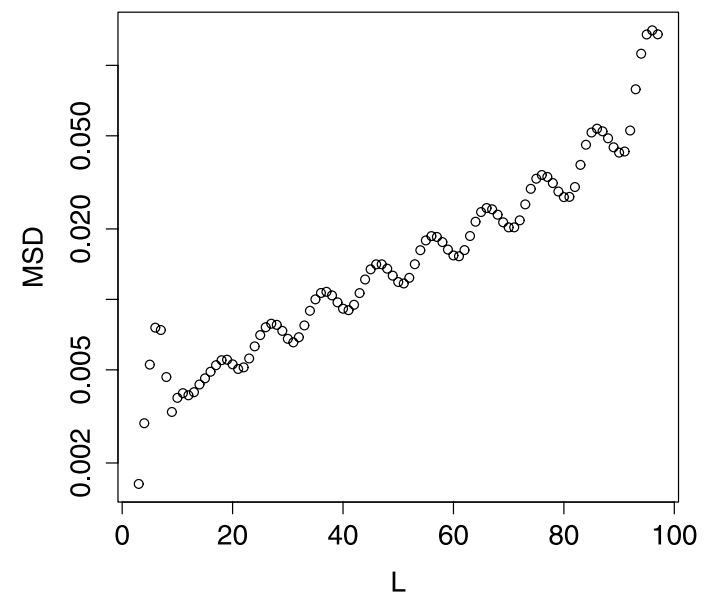

Figure 5. MSD of projector estimates: mixed residuals, t.s. (5) (log-scale).

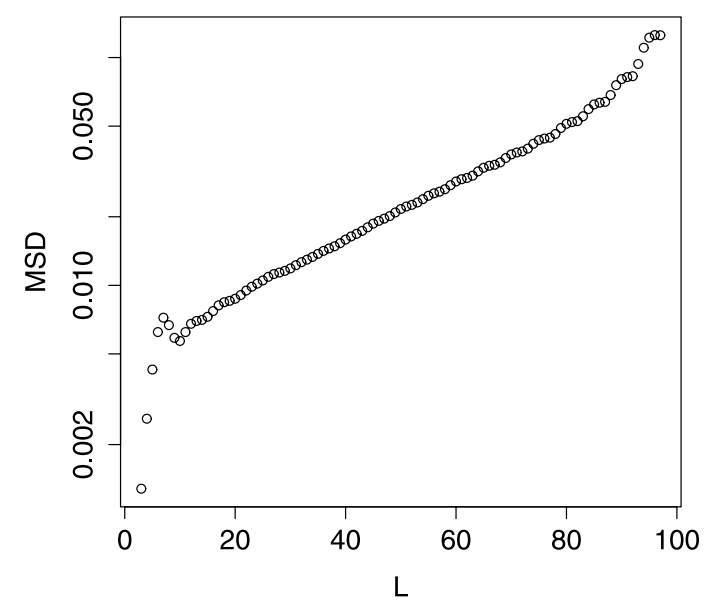

Figure 6. MSD of projector estimates: red-noise residuals, t.s. (6) (log-scale).

pure random and deterministic residuals. Below we show that this feature is valid for other kinds of problems.

To show that the fact that the noise is red (rather than white) does not interfere with the extraction of the signal, we consider the time series (6) with a red noise, see Fig. 6. Fig. 7 compares MSD for different structures of residuals (recall that SNR is the same). One can see that a red noise yields a slightly worse accuracy. Errors for the time series (5) lie between those for the time series (3) and the time series (4).

To summarize, in this section we demonstrated the behavior of errors of projector estimates for different types of residuals. However, we are usually interested in certain features of the signal space, rather than in the projector itself. Therefore, the results of this section provide just the basic information, which can be used for explanation of further results.

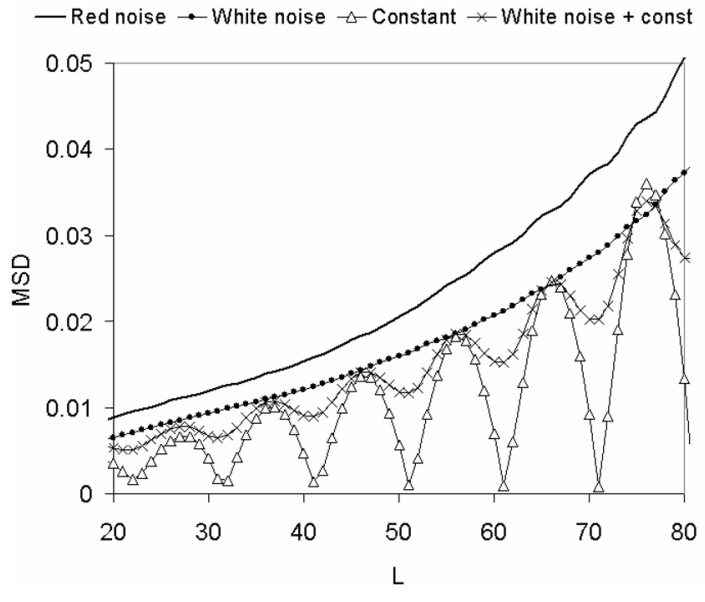

Figure 7. MSD of projector estimates: different types of residuals, t.s. (3) $-(6)$.

\section{SIGNAL EXTRACTION}

Recall that the reconstructed signal is obtained by applying the diagonal averaging to the reconstructed matrix calculated by the formula $\widetilde{\mathbf{S}}=\mathbf{U}_{r} \boldsymbol{\Lambda}_{r}^{1 / 2} \mathbf{V}_{r}^{\mathrm{T}}$, where $\mathbf{U}_{r}=\left[U_{1}\right.$ : $\left.\ldots: U_{r}\right], \mathbf{V}_{r}=\left[V_{1}: \ldots: V_{r}\right]$, and $\boldsymbol{\Lambda}_{r}=\operatorname{diag}\left(\lambda_{1}, \ldots, \lambda_{r}\right) ; U_{i}$, $V_{i}$ and $\lambda_{i}$ are defined in (1). Note that the columns of the matrix $\mathbf{U}_{r}$ form a basis of the perturbed signal subspace for the window length $L$ while the columns of $\mathbf{V}_{r}$ form a basis of the perturbed signal subspace for the window length $N-L+1$. First, this means that the results are the same for the window lengths $L$ and $N-L+1$. Then, as is shown in Figures 1-7, the signal-subspace perturbation grows as $L$ increases and decreases as $N-L+1$ increases. Thus, the resultant errors are caused by these contradictory tendencies. Fig. 4 demonstrates that the growth rate of errors in the projector estimation is larger for large $L>N / 2$. Therefore, there is no surprise that the reconstruction errors are large for small window lengths due to large errors in $\mathbf{V}_{r}$. However, the question about the optimal window lengths remains open.

Further we consider the dependence of the reconstruction error $\widetilde{s}_{l}-s_{l}$ on the window length in several examples. Note that an explicit asymptotic form is known for the example of noisy constant signal with $s_{n} \equiv c$ [17]. The paper [17] contains an explicit expression for the variance of the firstorder reconstruction errors, where the first order is given with respect to the perturbation of the signal by $R_{N}$ and not with respect to $N$. Strictly speaking, it is not necessary for the first-order error to be the main term of the error as $N \rightarrow \infty$. It has been checked by computer simulations that in the case of pure random noise we can consider the first-order error as the main error term (it is not true in the general case, see [27]). Computer simulations confirm that the qualitative results for the constant signal are valid for many other types of signals as well, including oscillations. 
To describe these results let us present the formula for the dependence of the asymptotic errors on the window length for a constant signal [17].

Let the window length $L \sim \beta N, 0<\beta \leq 1 / 2$, and $l$ be the index of the time series point, $l \sim \gamma N / 2,0 \leq \gamma \leq 1$, as $N \rightarrow \infty$. The value $\gamma=1$ corresponds to the middle of the time series; consequently, we present the formula for the first half of the time series with window lengths smaller than one-half of the time series length. Then the variance of the first-order errors has the following asymptotic form:

$$
\mathbb{D} s_{l}^{(1)} \sim \frac{\sigma^{2}}{N} \begin{cases}D_{1}(\beta, \gamma), & 0 \leq \gamma \leq 2 \min (\beta, 1-2 \beta) \\ D_{2}(\beta, \gamma), & 2 \min (\beta, 1-2 \beta)<\gamma<2 \beta \\ D_{3}(\beta, \gamma), & 2 \beta \leq \gamma \leq 1,\end{cases}
$$

as $N \rightarrow \infty$, where

$$
\begin{aligned}
D_{1}(\beta, \gamma)= & \frac{1}{12 \beta^{2}(1-\beta)^{2}}\left(\gamma^{2}(1+\beta)-2 \gamma \beta(1+\beta)^{2}\right. \\
& \left.+4 \beta\left(3-3 \beta+2 \beta^{2}\right)\right), \\
D_{2}(\beta, \gamma)= & \frac{1}{6 \beta^{2}(1-\beta)^{2} \gamma^{2}}\left(\gamma^{4}+2 \gamma^{3}\left(3 \beta-2-3 \beta^{2}\right)\right. \\
& +2 \gamma^{2}\left(3-9 \beta+12 \beta^{2}-4 \beta^{3}\right) \\
& +4 \gamma\left(-1+4 \beta-3 \beta^{2}-4 \beta^{3}+4 \beta^{4}\right) \\
& \left.+\left(8 \beta-56 \beta^{2}+144 \beta^{3}-160 \beta^{4}+64 \beta^{5}\right)\right), \\
D_{3}(\beta, \gamma)= & \frac{2}{3 \beta} .
\end{aligned}
$$

The points of change between the cases in (7) correspond to $l=K-L$ (i.e., $\gamma=2(1-2 \beta))$ and $l=L(\gamma=2 \beta)$. The former point of change is present if $K<2 L(\beta>1 / 3)$. Note that formula (7) can be extended to the window lengths $2<L<N-1(0<\beta<1)$ and to the indices of time series points $0 \leq l \leq N-1(0 \leq \gamma \leq 2)$ due to the symmetry of errors with respect to the middle of the time series and by the equivalence of results under the substitution of $L$ for $K$ $(\beta \leftrightarrow 1-\beta)$.

When we solve the problem of minimizing RMSE of estimation of $s_{l}$ at a fixed point $l$, the optimal window length varies from $N / 3$ to $N / 2$, see [17]. This means that even in the case with a constant signal the optimal window length, which minimizes the reconstruction errors as a whole, depends on the importance (weights) of each point of the time series. In any case, the general recommendation is to choose the window length slightly less than one-half of the time series length $N$. Note that the optimal window length provides a considerable improvement in the error rate (with respect to the choice $L=N / 2$ ) at the edge time series points, that is, for $l / N \approx 0$.

It has been shown for the projectors in Section 2 that for a noisy sine-wave signal (i.e. if the residuals do not contain a deterministic component), the divisibility of the

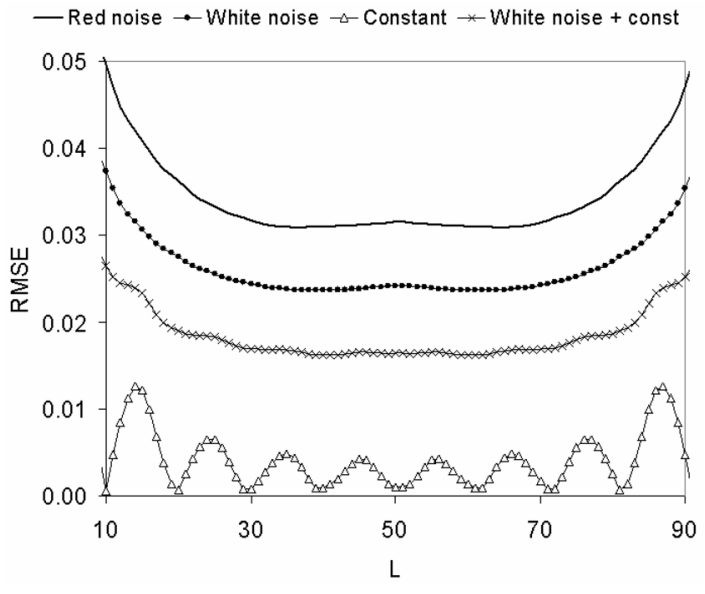

Figure 8. RMSE of signal estimates: different types of residuals, t.s. (3)-(6).

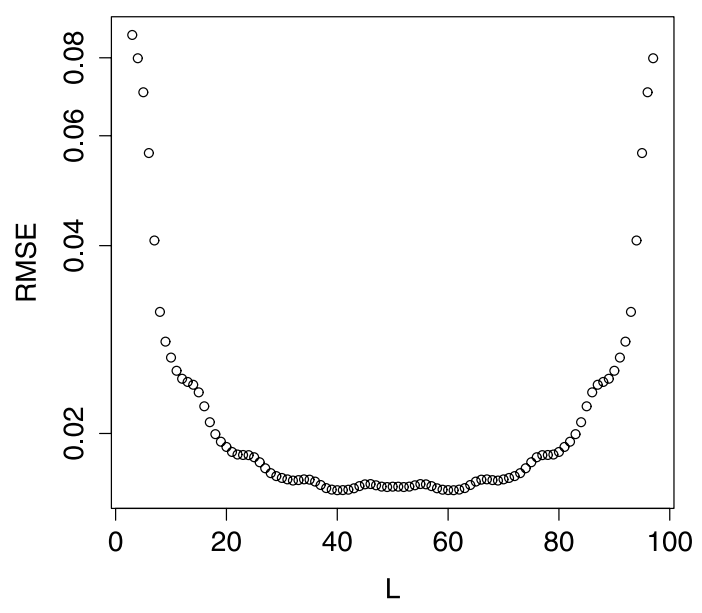

Figure 9. RMSE of signal estimates: mixed residuals, t.s. (5).

window length by the sine-wave period is not an important issue. The presence of a deterministic component in the residual makes this divisibility important. A similar effect takes place for the reconstruction errors $\left\|\widetilde{S}_{N}-S_{N}\right\|$, see Fig. 8 for the time series (3)-(6) with the same parameters.

To study the influence of the window length on RMSE, we consider RMSE for the reconstruction of the ten last points of the signal. Comparison of Fig. 9 and Fig. 10 shows that the impact of the divisibility of the window length by the sine-wave period is stronger for the edge points.

In Fig. 8 we observe that the optimal window length is close to $0.4 \mathrm{~N}$ in the case of random residual. However, the divisibility of the window length by the period (if the residuals contain a deterministic component) can be more important than the adjustment, e.g., than the transition from $N / 2$ to $0.4 N$. 


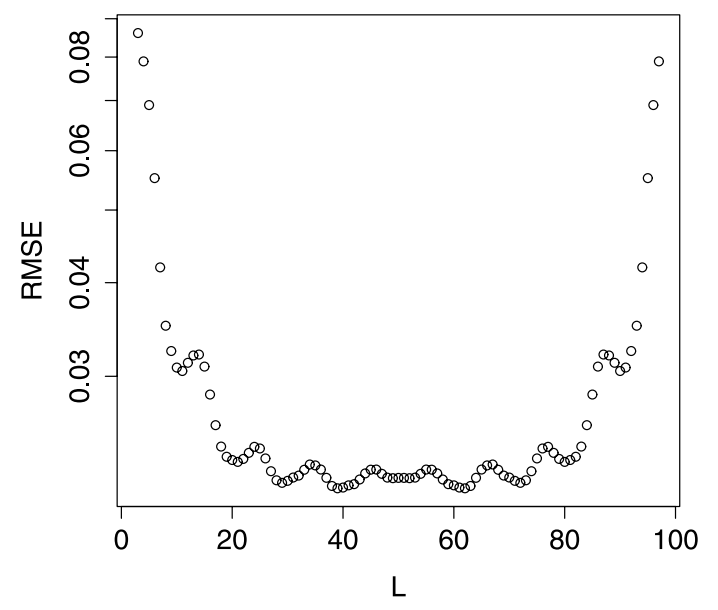

Figure 10. RMSE of signal estimates for the last 10 points: mixed residuals, t.s. (5).

\section{RECURRENT SSA FORECAST}

\subsection{Theory}

Let us consider the algorithm of recurrent forecasting [15] from the viewpoint of its connection to the signal subspace estimation. We start with some definitions.

Definition 4.1. A time series $S_{N}=\left\{s_{i}\right\}_{i=0}^{N-1}$ is governed by a linear recurrent formula (LRF), if there exist $a_{1}, \ldots, a_{t}$ such that

$$
s_{i+t}=\sum_{k=1}^{t} a_{k} s_{i+t-k}, \quad 0 \leq i<N-t, a_{t} \neq 0 .
$$

The number $t$ is called the order of the LRF, $a_{1}, \ldots, a_{t}$ are the coefficients of the LRF. If $t$ is the minimal order of an LRF that governs the time series $S_{N}$, then the corresponding LRF is called minimal.

Note that if the minimal LRF governing the signal $S_{N}$ has order $r$, then $S_{N}$ has finite rank $r$.

Definition 4.2. A polynomial $P_{t}(\mu)=\mu^{t}-\sum_{k=1}^{t} a_{k} \mu^{t-k}$ is called a characteristic polynomial of the LRF (8).

Let the time series $S=\left(s_{0}, \ldots, s_{n}, \ldots\right)$ satisfy the LRF (8) with $a_{t} \neq 0$ and $i \geq 0$. Consider the characteristic polynomial of the LRF (8) and denote the multiplicities of its (in general, complex) different roots $\mu_{1}, \ldots, \mu_{p}$ by $k_{m}$, where $1 \leq m \leq p, k_{1}+\cdots+k_{p}=t$. Note that the polynomial roots are non-zero as $a_{t} \neq 0$. Then the following well-known result (see e.g. [15, 18]) provides an explicit form for the series which satisfies the LRF.

Theorem 4.1. The time series $S=\left(s_{0}, \ldots, s_{n}, \ldots\right)$ satisfies the LRF (8) for all $i \geq 0$ iff

$$
s_{n}=\sum_{m=1}^{p}\left(\sum_{j=0}^{k_{m}-1} c_{m j} n^{j}\right) \mu_{m}^{n}
$$

where the complex coefficients $c_{m j}$ depend on the first $t$ points $s_{0}, \ldots, s_{t-1}$.

Note that if the LRF is not minimal, then the corresponding characteristic polynomial has extraneous roots. The extraneous roots do not affect the time series behavior, since the coefficients $c_{m j}$ for the corresponding summands are equal to zero. However, if one applies the LRF to the perturbed initial terms $\widetilde{s}_{0}, \ldots, \widetilde{s}_{t-1}$, then the extraneous roots start to affect the forecasting results. Therefore, the extraneous roots with modules greater than 1 are the most hazardous, since the extraneous summand $\mu^{n}$, caused by an extraneous root $\mu,|\mu|>1$, grows to infinity.

Unfortunately, if one analyzes/forecasts a real-life time series, the minimal LRF cannot be estimated with an appropriate accuracy and hence the presence of extraneous roots should be taken into account. The estimated LRF can be used both to find a parametric form of the signal (then we should remove extraneous roots) and also to forecast the time series (then we do not need to know the values of the polynomial roots; however, we would like to have no extraneous roots beyond the unit circle).

Finding the LRF Let $P_{1} \ldots P_{r}$ be an orthonormal basis of the signal subspace $\mathcal{L}_{r}^{(\mathrm{s})}=\operatorname{span}\left\{P_{1} \ldots P_{r}\right\}$ and $\mathcal{L}_{r+1, L}^{(\mathrm{s})}=$ $\operatorname{span}\left\{P_{r+1} \ldots P_{L}\right\}$ be its orthogonal complement. Denote $A=\left(a_{L-1}, \ldots, a_{1},-1\right)^{\mathrm{T}} \in \mathcal{L}_{r+1, L}^{(\mathrm{s})}, a_{L-1} \neq 0$. Then the time series satisfies the LRF $s_{n}=\sum_{k=1}^{L-1} a_{k} s_{n-k}, n=$ $L-1, \ldots, N-1$.

Conversely, if a time series is governed by an LRF, then the LRF's coefficients $B=\left(a_{L-1}, \ldots, a_{1}\right)^{\mathrm{T}}$ complemented with -1 yield the vector $\left(\begin{array}{c}B \\ -1\end{array}\right) \in \mathcal{L}_{r+1, L}^{(\mathrm{s})}$. Any LRF that governs the time series can be treated as a forward linear prediction. In addition, if we consider a vector from $\mathcal{L}_{r+1, L}^{(\mathrm{s})}$ with -1 as the first coordinate, then we obtain the so-called backward linear prediction [33].

Let us denote the matrix $\mathbf{A}$ without the last row by $\underline{\mathbf{A}}$ and the matrix $\mathbf{A}$ without the first row by $\overline{\mathbf{A}}$.

From the viewpoint of prediction, the LRF governing a time series of rank $r$ has coefficients derived from the condition $\underline{\mathbf{S}}^{\mathrm{T}} B=\left(s_{L-1}, \ldots, s_{N-1}\right)^{\mathrm{T}}$. This system of linear equations may have several solutions, since the vector $\left(s_{L-1}, \ldots, s_{N-1}\right)^{\mathrm{T}}$ belongs to the column space of the matrix $\underline{\mathbf{S}}^{\mathrm{T}}$. It is well-known that the least-squares solution expressed by the pseudo-inverse to $\underline{\mathbf{S}}^{\mathrm{T}}$ yields the vector $B$ with the minimum norm (the TLS-solution coincides with it).

It can be shown that the minimum-norm solution $B_{\mathrm{LS}}$ can be expressed as

$$
B_{\mathrm{LS}}=\left(a_{L-1}, \ldots, a_{1}\right)^{\mathrm{T}}=\frac{1}{1-\nu^{2}} \sum_{i=1}^{r} \pi_{i} \underline{P_{i}},
$$

where $\pi_{i}$ is the last coordinates of $P_{i}$ and $\nu^{2}=\sum_{i=1}^{r} \pi_{i}^{2}$.

Thus, one of the vectors from $\mathcal{L}_{r+1, L}^{(\mathrm{s})}$, which equals $A_{\mathrm{LS}}=$ $\left(\begin{array}{c}B_{\mathrm{LS}} \\ -1\end{array}\right)$, has a special significance and is called the min-norm 
(forward) prediction. Similarly, we can obtain a formula for the min-norm backward prediction.

It is shown in $[15,23]$ that the forward min-norm prediction vector $A_{\mathrm{LS}}$ is the normalized (so that its last coordinate is equal to -1$)$ projection of $\mathbf{e}_{L}=\left(\mathbf{0}_{L-1}, 1\right)^{\mathrm{T}}$ on the orthogonal complement to the signal subspace. Therefore, the min-norm prediction vector depends on the signal subspace only.

The paper [24] contains a property of the min-norm LRF, which is very important for forecasting: all the extraneous roots of the min-norm LRF lie inside the unit circle on the complex plane. This gives us the hope that in the case of real-life time series (when the min-norm LRF and the related initial data are perturbed) the extraneous summands in (9) decrease and just slightly influence the forecast. Moreover, in view of results about the distribution of extraneous roots (see [28, 34]), we can suppose that the extraneous summands are able to compensate one another.

Note that this min-norm LRF forms the basis for the forecasting methods introduced in [15]. In particular, the recurrent forecast is constructed by applying formula (10) with $P_{i}=U_{i}$, where $U_{i}$ are taken from (1), to the last $L-1$ reconstructed signal points $\widetilde{s}_{N-(L-1)}, \ldots, \widetilde{s}_{N-1}$.

\subsection{Dependence on the window length}

Let us consider the dependence of the forecast accuracy on the window length $L$ (we consider the signal rank $r$ as given in advance). Note that the forecasting procedure uses two objects: the LRF itself and the initial data for this LRF taken from the last points of the reconstructed signal $\widetilde{S}_{N}$. Let us denote the vector constructed from the $L-1$ last signal points by $V$ and the vector constructed from the $L-1$ last reconstructed (i.e., perturbed) signal points by $V+\Delta V$. Likewise, we denote the vector of coefficients of the true min-norm LRF by $A$ and the vector of the estimated LRF coefficients by $A+\Delta A$. Then the forecast error is $A^{\mathrm{T}} \Delta V+$ $(\Delta A)^{\mathrm{T}} V+(\Delta A)^{\mathrm{T}} \Delta V$.

Therefore, the first-order error consists of two kinds of errors:

1. the errors in the LRF coefficients that are caused by an error of the projection onto the signal subspace $(\Delta A)^{\mathrm{T}} V$;

2. the errors of signal reconstruction $A^{\mathrm{T}} \Delta V$.

Let us investigate these two error sources separately. To do this, we apply the LRF that was estimated with the window length $L_{\mathrm{LRF}}$ to the true signal values and apply the true LRF to the estimated signal values that were reconstructed with the window length $L_{\text {rec }}$.

We consider the time series (4) with $\sigma=0.1, N=399$ and $\ln b=0,0.01,-0.01$. Estimation (by 1000 simulations) of RMSE of the one-term ahead forecast is depicted in Fig. 11 for $L_{\mathrm{rec}}=200$. Values of $L_{\mathrm{LRF}}$ are varied from 20 to 380 with increment 20 . Similar graphs for different $L_{\mathrm{rec}}$ are

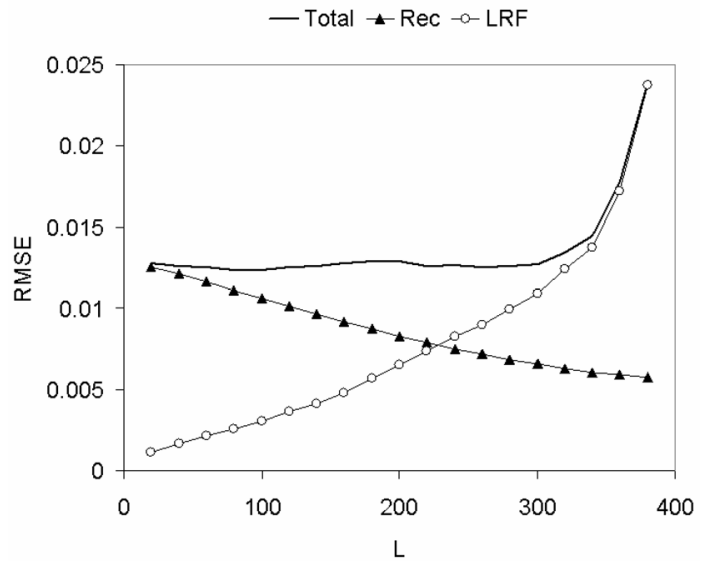

Figure 11. RMSE of forecast as a function of $L_{\mathrm{LRF}}$ : t.s. (4) with $b=1, L_{\mathrm{rec}}=200$.

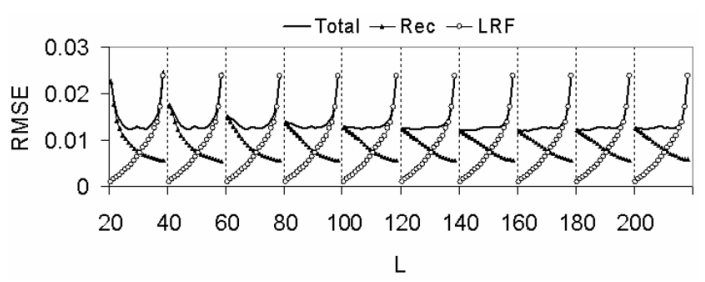

Figure 12. RMSE of forecast: t.s. (4) with $b=1$, different $L_{\text {rec. }}$.

presented in Fig. 12. The labels of x-axis correspond to the values of $L_{\mathrm{rec}}$.

The line marked 'total' shows the accuracy (RMSE) of the forecast with the corresponding window lengths. The line marked 'LRF' corresponds to $(\Delta A)^{\mathrm{T}} V$. The 'LRF' errors look like the errors of the estimator $\mathrm{P}_{r}$ of the projector on the signal space (see Fig. 4). This is not surprising since the coefficients of the LRF are proportional to the projection $\mathbf{U}_{r+1, L} \mathbf{U}_{r+1, L}^{\mathrm{T}} \mathbf{e}_{L}=\mathrm{P}_{r}^{\perp} \mathbf{e}_{L}$. Naturally, the 'LRF' errors do not depend on the window length $L_{\text {rec }}$ used for reconstruction. The typical behavior of this part of the error is as follows: the larger the window length, the larger the error.

The line marked 'Rec' corresponds to $A^{\mathrm{T}} \Delta V$. We can see that the larger the window length, the smaller the error. This can be interpreted in the following way: extraneous roots that are located closely to the uniform distribution on a circle compensate one another (see [28, 34] for several results of this kind).

Figures 11-12 show that the accuracy of forecasts is stable within a wide range of window lengths. In particular, $L_{\mathrm{LRF}}$ and $L_{\text {rec }}$ slightly smaller than $N / 2$ are quite appropriate. Also, we can take either small $L_{\mathrm{LRF}}$ and $L_{\text {rec }} \sim N / 2$ or $L_{\mathrm{LRF}} \sim N / 2$ and small $L_{\mathrm{rec}}$. The former is the preferred choice, since the errors are smaller and more robust to the changes in the window length, see Fig. 12. 


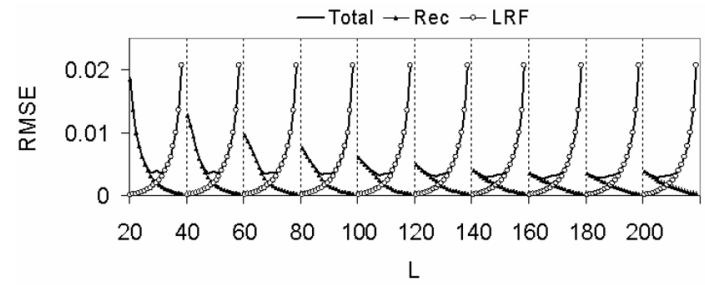

Figure 13. RMSE of forecast: t.s. (4) with $b<1$, different $L_{\text {rec }}$.

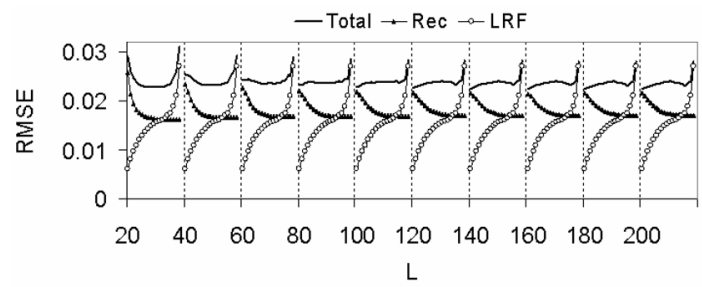

Figure 14. RMSE of forecast: t.s. (4) with $b>1$, different $L_{\text {rec }}$.

Recommendations on the window length choice naturally depend on the forms of the signal and the residual. Figures 13 and 14 contain RMSE for damped sine waves. The interpretation of figures and the RMSE behavior are similar.

The deterministic residual can provide a specific behavior of errors. Specifically, for the time series (2) we obtain that the choice of an even $N$ and odd values of $L_{\mathrm{LRF}}$ provides a decrease of the errors of projectors as the window length increases (see Fig. 1). Therefore, for such choice of $L$ and $N$ we observe similar behavior for two sources of forecast errors and the optimal choice is that the window length should be as large as possible.

\section{SUBSPACE-BASED METHODS OF PARAMETER ESTIMATION}

While the problems of reconstruction and forecasting are traditionally included into the scope of problems solved by SSA, estimation of signal parameters by the subspace-based methods is often not considered within the framework of SSA. Therefore, we will describe the subspace-based methods in more detail to demonstrate their cohesion with SSA.

\subsection{Basic facts}

Definition 5.1. The companion matrix of a polynomial $p(\mu)=\mu^{n}+c_{1} \mu^{n-1}+\cdots+c_{n-1} \mu+c_{n}$ is

$$
\mathbf{C}=\left(\begin{array}{lllll}
0 & 0 & 0 & \ldots & -c_{n} \\
1 & 0 & 0 & \ldots & -c_{n-1} \\
0 & 1 & \ldots & 0 & -c_{n-2} \\
\vdots & \vdots & \ddots & \vdots & \vdots \\
0 & 0 & \ldots & 1 & -c_{1}
\end{array}\right)
$$

Proposition 5.1. The roots of a polynomial coincide with the eigenvalues of its companion matrix.

Note that the multiplicities of roots are equal to the algebraic multiplicities of the eigenvalues of the companion matrix (i.e., to the multiplicities of roots of the matrix characteristic polynomial). However, these multiplicities do not always coincide with the geometric multiplicities equal to the dimensions of the eigenspaces corresponding to the eigenvalues.

To derive the analytic form (9) of the signal we need to find roots of the characteristic polynomial of the LRF which governs the signal. By Proposition 5.1, we have to find either the roots of the characteristic polynomial or the eigenvalues of its companion matrix. The latter does not require a linear recurrent formula itself. Let us demonstrate that to find the signal roots it is sufficient to know the basis of the signal trajectory space.

Let $\mathbf{C}$ be a full-rank $d \times d$ matrix, $X \in \mathbb{R}^{d}$, and $\mathbf{X}$ be a full-rank $L \times d$ matrix, $L>d$, which can be expressed as

$$
\mathbf{X}=\left(\begin{array}{l}
X^{\mathrm{T}} \\
X^{\mathrm{T}} \mathbf{C} \\
\vdots \\
X^{\mathrm{T}} \mathbf{C}^{L-1}
\end{array}\right)
$$

Let us again denote the matrix $\mathbf{X}$ without the last row by $\underline{\mathbf{X}}$ and the matrix $\mathbf{X}$ without its first row by $\overline{\mathbf{X}}$. It is clear that $\overline{\mathbf{X}}=\underline{\mathbf{X}} \mathbf{C}$. We call this property of $\mathbf{X}$ shift property given by the matrix $\mathbf{C}$.

Proposition 5.2. Let $\mathbf{X}$ satisfy the shift property given by the matrix $\mathbf{C}, \mathbf{P}$ be a full-rank $d \times d$ matrix, and $\mathbf{Y}=\mathbf{X P}$. Then the matrix $\mathbf{Y}$ satisfies the shift property given by the matrix $\mathbf{D}=\mathbf{P}^{-1} \mathbf{C P}$, i.e., $\overline{\mathbf{Y}}=\underline{\mathbf{Y}} \mathbf{D}$.

Proof.

$$
\begin{aligned}
\mathbf{X P} & =\left(\begin{array}{l}
X^{\mathrm{T}} \mathbf{P} \\
X^{\mathrm{T}} \mathbf{C} \mathbf{P} \\
\vdots \\
X^{\mathrm{T}} \mathbf{C}^{L-1} \mathbf{P}
\end{array}\right)=\left(\begin{array}{l}
X^{\mathrm{T}} \mathbf{P} \\
X^{\mathrm{T}} \mathbf{P}\left(\mathbf{P}^{-1} \mathbf{C P}\right) \\
\vdots \\
X^{\mathrm{T}} \mathbf{P}\left(\mathbf{P}^{-1} \mathbf{C}^{L-1} \mathbf{P}\right)
\end{array}\right) \\
& =\left(\begin{array}{l}
X^{\mathrm{T}} \mathbf{P} \\
X^{\mathrm{T}} \mathbf{P} \mathbf{D} \\
\vdots \\
X^{\mathrm{T}} \mathbf{P D}^{L-1}
\end{array}\right)=\left(\begin{array}{l}
Y^{\mathrm{T}} \\
Y^{\mathrm{T}} \mathbf{D} \\
\vdots \\
Y^{\mathrm{T}} \mathbf{D}^{L-1}
\end{array}\right)
\end{aligned}
$$

where $Y=X^{\mathrm{T}} \mathbf{P}$. This implies the shift property for $\mathbf{Y}$.

Note that multiplication by a nonsingular matrix $\mathbf{P}$ can be considered as a change of coordinates in the column space of the matrix $\mathbf{X}$.

It is easily seen that the matrices $\mathbf{C}$ and $\mathbf{D}=\mathbf{P}^{-1} \mathbf{C P}$ have the same eigenvalues; these matrices are called similar. 
Remark 5.1. Let the matrix $\mathbf{Y}$ satisfy the shift property given by the matrix $\mathbf{D}$. Then $\mathbf{D}=\underline{\mathbf{Y}}^{\dagger} \overline{\mathbf{Y}}$, where $\mathbf{A}^{\dagger}$ denotes the Moore-Penrose pseudoinverse of $\mathbf{A}$.

Proposition 5.3. Let a time series $F$ satisfy the minimal $L R F$ (8) of order $d, L \geq d$ be the window length, $\mathbf{C}$ be the companion matrix of the characteristic polynomial of this $L R F$. Then any $L \times d$ matrix $\mathbf{Y}$ with columns forming a basis of the trajectory space of $F$ satisfies the shift property given by some matrix D. Moreover, the eigenvalues of this shift matrix $\mathbf{D}$ coincide with the eigenvalues of the companion matrix $\mathbf{C}$ and, therefore, with the roots of the characteristic polynomial of the LRF.

Proof. Note that for any $0 \leq i<N-1$ we have

$$
\left(f_{i}, f_{i+1}, \ldots, f_{i+(d-1)}\right) \mathbf{C}=\left(f_{i+1}, f_{i+2}, \ldots, f_{i+d}\right) .
$$

Therefore, (11) holds for $X=\left(f_{0}, f_{1}, \ldots, f_{d-1}\right)^{\mathrm{T}}$. It is known that for a time series governed by a minimal LRF of order $d$, any $d$ adjacent vectors of the embedding are independent. Consequently, the matrix $\mathbf{X}$ is of full rank and we can apply Proposition 5.2.

Remark 5.2. The SVD of the $L$-trajectory matrix of a time series provides a basis of its trajectory space. Namely, the left singular vectors which correspond to the nonzero singular values form such a basis. If we observe a time series 'signal + residual', then the SVD of its $L$-trajectory matrix provides the basis of the signal subspace under the condition of exact strong separability of the signal and the residual, see [15].

\subsection{ESPRIT}

Consider a time series $F_{N}=\left\{f_{i}\right\}_{i=0}^{N-1}, f_{i}=s_{i}+r_{i}$, where $S_{N}=\left\{s_{i}\right\}_{i=0}^{N-1}$ is a time series governed by a linear recurrent formula of order $r$ (i.e. signal), $R_{N}=\left\{r_{i}\right\}_{i=0}^{N-1}$ is a residual (noise, perturbation). Let again $\mathbf{X}$ be the trajectory matrix of $F_{N}$. In the case of exact or approximate separability of the signal and the residual and if the signal is dominant, the subspace $\operatorname{span}\left\{U_{1}, \ldots, U_{r}\right\}$ can be considered as an estimate of the true signal subspace. Therefore, we can use $\widetilde{\mathbf{Y}}=\mathbf{U}_{r}=$ $\left[U_{1}: \ldots: U_{r}\right]$ as an estimate of $\mathbf{Y}$ from Proposition 5.3. Then the shift property is fulfilled only approximately and $\underline{\mathbf{U}_{r}} \mathbf{D} \approx \overline{\mathbf{U}_{r}}$.

Let us study the methods of finding the matrix $\mathbf{D}$. The idea was introduced in [25] devoted to the problem of estimating the frequencies in a sum of sinusoids, in the presence of noise. The method was given its well-known name ESPRIT in [30], which was later used in many other papers devoted to the DOA (Direction of Arrival) problem. This method is also called Hankel SVD (HSVD [3], for the LS version) and Hankel Total Least Squares (HTLS [35], for the TLS version).

\subsubsection{Least Squares (LS-ESPRIT), HSVD}

The LS-ESPRIT estimate of the matrix $\mathbf{D}$ is

$$
\widehat{\mathbf{D}}=\underline{\mathbf{U}}_{r}^{\dagger} \overline{\mathbf{U}}_{r}=\left({\underline{\mathbf{U}_{r}}}^{\mathrm{T}} \underline{\mathbf{U}_{r}}\right)^{-1} \underline{\mathbf{U}}_{r}^{\mathrm{T}} \overline{\mathbf{U}_{r}} .
$$

The eigenvalues of $\widehat{\mathbf{D}}$ do not depend on the choice of the basis of the subspace $\operatorname{span}\left\{U_{1}, \ldots, U_{r}\right\}$. In fact, if we change the coordinates so that $\mathbf{W}=\mathbf{U}_{r} \mathbf{P}$, where $\mathbf{P}$ is a nondegenerate $r \times r$ transfer matrix, then $\underline{\mathbf{W}}=\mathbf{U}_{r} \mathbf{P}$ and $\overline{\mathbf{W}}=\overline{\mathbf{U}_{r}} \mathbf{P}$. Hence, by the direct substitution we have that the estimators of $\mathbf{D}$ obtained by using $\mathbf{W}$ or $\mathbf{U}_{r}$ are similar matrices and therefore have the same eigenvalues.

\subsubsection{Total Least Squares (TLS-ESPRIT), HTLS}

Since $\mathbf{U}_{r}$ is known only approximately, then there are errors in both $\underline{\mathbf{U}_{r}}$ and $\overline{\mathbf{U}_{r}}$. Therefore, the solution of the approximate equality $\underline{\mathbf{U}_{r}} \mathbf{D} \approx \overline{\mathbf{U}_{r}}$ based on the Total Least Squares method can be more accurate.

Let us recall that to solve the equation $\mathbf{A X} \approx \mathbf{B}$ TLS minimizes the following sum:

$$
\begin{gathered}
\|\widetilde{\mathbf{A}}-\mathbf{A}\|_{\mathrm{F}}^{2}+\|\widetilde{\mathbf{B}}-\mathbf{B}\|_{\mathrm{F}}^{2} \longrightarrow \text { min, where } \\
(\widetilde{\mathbf{A}}, \widetilde{\mathbf{B}}) \in\{(\widetilde{\mathbf{A}}, \widetilde{\mathbf{B}}): \exists \mathbf{Z}, \widetilde{\mathbf{A}} \mathbf{Z}=\widetilde{\mathbf{B}}\} .
\end{gathered}
$$

Set $\mathbf{A}=\underline{\mathbf{U}_{r}}, \mathbf{B}=\overline{\mathbf{U}_{r}}$ in (13). Then the matrix $\mathbf{Z}$ that minimizes (13) is called the TLS-estimate of $\mathbf{D}$ (see [8] for explicit formulas).

Let us consider the dependence of the TLS-ESPRIT solution on the choice of the basis of $\operatorname{span}\left\{U_{1}, \ldots, U_{r}\right\}$. Generally speaking (computer experiments confirm this), this dependence takes place. However, the following assertion can be proved.

Proposition 5.4. The TLS-ESPRIT solution does not depend on the unitary transformation of the basis of $\operatorname{span}\left\{U_{1} \ldots U_{r}\right\}$. In particular, the TLS-ESPRIT estimate is the same for any orthonormal basis.

Indeed, suppose that the minimum in (13) is achieved at some $\mathbf{Z}$. Let us consider the revised problem (13) with replacement of $\mathbf{A}$ and $\mathbf{B}$ by $\mathbf{A P}$ and $\mathbf{B P}$, respectively. It is easy to see that if $\mathbf{P}$ is an $r \times r$ unitary matrix (and therefore preserves norms), then the solution of the revised problem (13) has the form of $\mathbf{P}^{-1} \mathbf{Z P}$, which is similar to $\mathbf{Z}$. Thus, the eigenvalues of the solution of the problem (13) do not depend on simultaneous rotations/reflections in the column spaces of the matrices $\mathbf{A}$ and $\mathbf{B}$.

\subsubsection{DOA and time series analysis}

Recall that ESPRIT-like methods can be applied both to the general problem of parameter estimation for the time series and to the DOA problem specifically. However, there are several special aspects of application to DOA:

1. Input data is in the form of an $L \times K$ matrix (an analogue to the trajectory matrix). The matrix satisfies the shift property but it is not necessarily a Hankel matrix. 
2. The number of sensors $(L)$ is fixed and is usually not large. Therefore, the properties of the method are considered under the condition that $L$ is fixed and $K \rightarrow \infty$.

3. The problem can be stated so that the entries of the input matrix are noisy with independent noise realizations (this does not hold for the trajectory matrices where both the signal and noise form Hankel matrices).

4. The data for DOA is mostly complex-valued with complex circular white Gaussian noise.

Generally, these aspects can influence the statement of the parameter choice problem and the rule of the optimal parameter choice.

\subsubsection{Dependence on the window length}

The paper [2] contains the following theoretical result: while estimating the frequency of a noisy sinusoid, the asymptotic $(N \rightarrow \infty)$ variance of the first-order error has order $1 /\left(K^{2} L\right)$ and is symmetric with respect to $N / 2$. Therefore, the asymptotic optimal window length is equal to $N / 3$ or $2 N / 3$. Numerical experiments confirm this conclusion. Let us remark that it is not always true that the first-order (with respect to the perturbation level) error is the mainterm error as the time series length tends to infinity. Therefore, it is better to check the correspondence between the first-order error and the total error through simulation.

In [9] an explicit form of the asymptotic variance of the first-order error is derived in the general case of damped complex exponentials. In the case of undamped complex exponentials, the derived form coincides with that in [2]. As for damped complex exponentials, the result is that the optimal window length lies between $N / 3$ and $N / 2$ and approaches $N / 2$ as the damping factor increases. It is shown in [9] that for $s_{n}=\exp ((\alpha+i \beta) n), i=\sqrt{-1}$, the first-order variances of the ESPRIT estimates of $\alpha$ and $\beta$ are equal. Therefore, the optimal window lengths are the same for estimators of the damping factor $\alpha$ and of the frequency $\beta$.

In the previous sections we demonstrated that the separability of the signal from deterministic and stochastic residuals has a different nature and therefore leads to different consequences. Let us consider how this difference reveals itself in the problem of the frequency estimation by ESPRIT. We perform simulations for the time series (3)-(6) with $c=\sigma=0.1, b=1, N=100$.

Fig. 15 contains the results for the deterministic perturbation, including the specific behavior of RMSE in the case of one-sided (left) orthogonality. Fig. 16 contains RMSE of frequency estimates for different kinds of residuals. The behavior of errors is very similar to that in the signal reconstruction, see Fig. 8. Also, the errors of frequency and exponential rate (damping factor) estimates are approximately equal if $L$ is proportional to $N$. The main difference from the reconstruction errors is in the size of errors, which is much smaller. Therefore we use 1000 instead of 100 simulated series to estimate RMSE with sufficient accuracy.

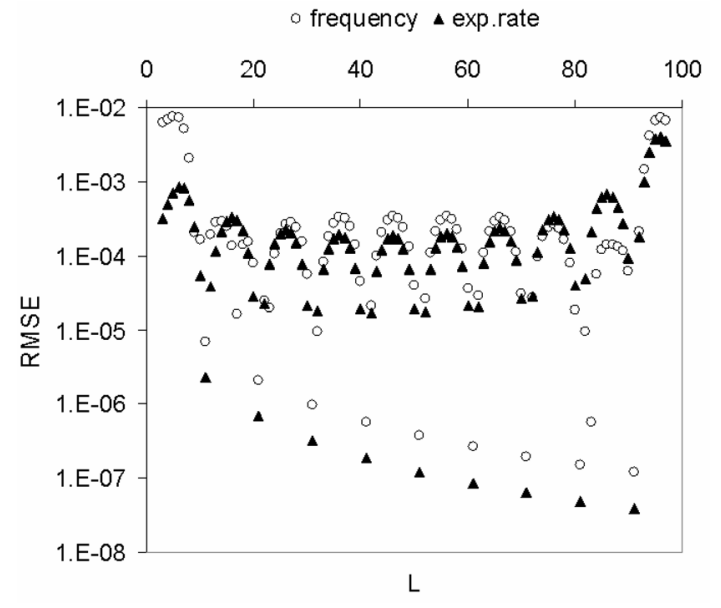

Figure 15. RMSE of frequency and exp.rate estimates: t.s. (3) (log-scale).

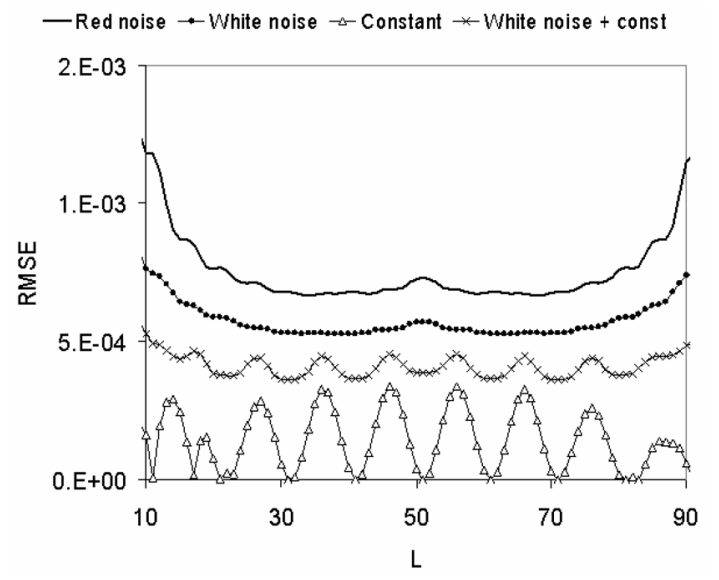

Figure 16. RMSE of frequency estimates: different types of residuals, t.s. (3)-(6), $L \sim N / 2$.

Fig. 17 focuses attention on the error behavior for small window lengths. One can see that the dominance of errors for the time series (3) with deterministic residuals is a distinctive feature.

\subsection{Brief review of other subspace-based methods}

In this subsection, we briefly describe several subspacebased methods in addition to ESPRIT-like ones. These methods are applied to time series governed by LRFs and in fact estimate the main (signal) roots of the corresponding characteristic polynomials. The basic subspace-based methods were developed for the case of a noisy sum of imaginary exponentials (cisoids) or of real sinusoids, for the purpose of frequency estimation, see e.g. [32]. We are mostly interested in the methods that can be applied to any time series of finite rank given in the form (9). 


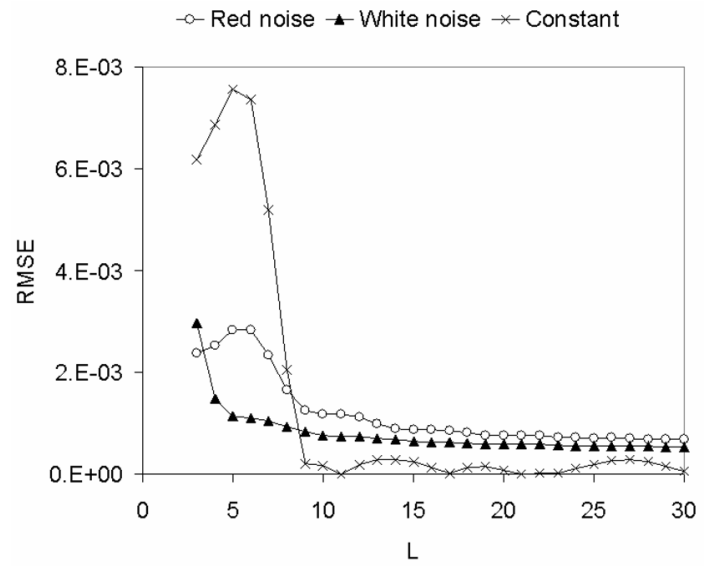

Figure 17. RMSE of frequency estimates: different types of residuals, t.s. (3) $-(6), L$ is small.

We start with the description of general methods in the complex-valued case.

Version 1 Consider an LRF that governs the signal (the best choice is the min-norm LRF, see Section 4.1, however this is not essential). Then we find all roots $\mu_{m}$ of the characteristic polynomial of this LRF and then find coefficients $c_{m j}$ in (9). The coefficients $c_{m j}$ corresponding to the extraneous roots are equal to 0 . In the case of a noisy signal, $\widehat{\mu}_{m}$ are the roots of a polynomial with coefficients from a vector that belongs to $\mathcal{L}_{r}^{\perp}$, and the extraneous roots have small absolute values of the LS estimates $\widehat{c}_{m j}$.

Version 2 Let us consider the forward and backward minnorm predictions. It is known that the corresponding characteristic polynomials have the conjugate extraneous roots and their signal roots are connected by the relation $z^{\prime}=$ $z^{*} /\|z\|^{2}$. Note that the forward prediction given by a vector $A \in \mathcal{L}_{r}^{\perp}$ corresponds to the roots of $\langle Z(z), A\rangle=0$, where $Z(z)=\left(1, z, \ldots, z^{L-1}\right)^{\mathrm{T}}$ and $\langle\cdot, \cdot\rangle$ is the inner product in $\mathbb{C}$. At the same time, the backward prediction given by a vector $B \in \mathcal{L}_{r}^{\perp}$ corresponds to the roots of $\langle Z(1 / z), B\rangle=0$. If we consider the roots of the forward and backward minnorm polynomials together, then all the extraneous roots lie inside the unit circle, while one of $z^{\prime}$ and $z$ is located on or beyond the unit circle. This allows us to detect the signal roots.

Version 3 Let us take a set of vectors from $\mathcal{L}_{r}^{\perp}$. Each vector from $\mathcal{L}_{r}^{\perp}$ with a nonzero last coordinate generates an LRF. The signal roots of the characteristic polynomials of these LRFs are equal (or are close for a noisy signal), whereas the extraneous roots are arbitrary. Therefore, the signal roots correspond to clusters of roots if we consider pooled roots. One of the ways to choose vectors from $\mathcal{L}_{r}^{\perp}$ is to take the set of eigenvectors corresponding to noise.

There are some other methods that are developed for estimating frequencies in a noisy sum of undamped sinu- soids or complex exponentials. Let for simplicity $s_{n}=$ $\sum_{k=1}^{r} c_{k} e^{2 \pi i \omega_{k} n}$. In this case, the signal roots $e^{2 \pi i \omega_{k}}$ have absolute values equal to 1 and can be parameterized only by one parameter (frequency). Let $W=W(\omega)=Z\left(e^{2 \pi i \omega}\right)$. Since $W\left(\omega_{k}\right) \in \mathcal{L}_{r}^{(\mathrm{s})},\left\langle W\left(\omega_{k}\right), A\right\rangle=0$ for all $A \in \mathcal{L}_{r+1, L}^{(\mathrm{s})}$. If $A \in \mathcal{L}_{r}^{\perp}$, then we can consider the square of the cosine of the angle between $W\left(\omega_{k}\right)$ and $A$ as a measure of their orthogonality. This idea forms the basis for the Min-Norm and MUSIC methods. The names of methods in which roots are ordered by the absolute value of the deviation of their modules from the unit circle begin with 'root-'.

Version 4. Min-Norm Let $f(\omega)=\cos ^{2}(\widehat{W(\omega)}, A)$, where $A=\mathrm{P}_{r}^{\perp} \mathbf{e}_{L} \in \mathcal{L}_{r}^{\perp}$ is the vector corresponding to the minnorm forward prediction. The Min-Norm method consists in searching for the maximums of $1 / f(\omega)$, and the function $1 / f(\omega)$ of $\omega$ is interpreted as a so-called pseudospectrum with peaks at the frequencies presented in the signal.

Version 5. Root Min-Norm We consider the min-norm LRF and choose the $r$ closest to the unit circle roots of its characteristic polynomial.

Version 6. MUSIC Let $f(\omega)=\cos ^{2}\left(\widehat{W(\omega), \mathcal{L}_{r}^{\perp}}\right)$. If we take eigenvectors $U_{j}, j=r+1, \ldots, L$, as a basis of $\mathcal{L}_{r}^{\perp}$, then $\mathbf{U}_{r+1, L} \mathbf{U}_{r+1, L}^{*}$ provides the matrix of projection on $\mathcal{L}_{r}^{\perp}$ and therefore $f(\omega)=W^{*}\left(\mathbf{U}_{r+1, L} \mathbf{U}_{r+1, L}^{*}\right) W /\|W\|^{2}=$ $\sum_{j=r+1}^{L} f_{j}(\omega)$, where $f_{j}(\omega)=\cos ^{2}\left(\widehat{W(\omega), U_{j}}\right)$. Thus, the MUSIC method can be considered from the viewpoint of the subspace properties and does not require the computation of roots of characteristic polynomials. Similar to the MinNorm method, the MUSIC method consists in searching for the maximums of the pseudospectrum $1 / f(\omega)$.

There is a modification of MUSIC called 'EV'. In this modification, the pseudospectrum is constructed on the base of the weighted sum $\sum_{j=r+1}^{L} f_{j}(\omega) / \lambda_{j}$. The EV method can improve frequency estimates if the signal rank $r$ is detected with error, since the weights decrease the contribution of summands corresponding to the noise eigenvectors that are adjacent to the signal ones. Note that the EV method is not expressed in terms of the signal subspace.

Version 7. Root-MUSIC This method involves calculation of the polynomial roots. Specifically, we calculate the roots of a polynomial of $z$ solving the equation $(Z(1 / z))^{\mathrm{T}} \mathbf{U}_{r+1, L} \mathbf{U}_{r+1, L}^{*} Z(z)=0$. This polynomial can be considered as the multiplication of polynomials with signal roots connected by the relation $z^{\prime}=z^{*} /\|z\|^{2}$, that is, the forward and backward predictions. Then the roots with modules less than or equal to 1 are assembled in the ascending order of their modules. The signal roots have modules that are the closest to 1.

\section{THE RATE OF CONVERGENCE}

The paper [27] contains theoretical results on convergence (as the time series length $N$ tends to infinity) for the methods that are based on the estimation of the signal subspace. 
Table 1. The rate of convergence of reconstruction: estimated $\Delta$

\begin{tabular}{ccccc}
\hline \hline$L$ & $\mathrm{c}$ & $\mathrm{c}+\mathrm{wn}$ & $\mathrm{wn}$ & $\mathrm{rn}$ \\
\hline$r+1$ & 1.0 & 1.0 & 1.0 & 1.0 \\
20 & - & 1.0 & 1.0 & 1.0 \\
25 & 1.0 & 1.0 & 1.0 & 1.0 \\
\hline$(N+1) / 2-5$ & 8.0 & 1.9 & 2.0 & 2.1 \\
$(N+1) / 2$ & - & 1.9 & 2.0 & 2.1 \\
\hline
\end{tabular}

Table 2. The rate of convergence of projector estimates: estimated $\Delta$

\begin{tabular}{ccccc}
\hline \hline$L$ & c & c+wn & wn & rn \\
\hline$r+1$ & 1.0 & 1.0 & 2.2 & 1.0 \\
20 & - & 3.1 & 2.8 & 1.1 \\
25 & 1.0 & 1.0 & 3.0 & 1.1 \\
\hline$(N+1) / 2-5$ & 4.0 & 2.0 & 2.0 & 2.0 \\
$(N+1) / 2$ & - & 2.0 & 2.0 & 2.0 \\
\hline
\end{tabular}

Here we investigate the rate of convergence by means of examples.

Let us consider two time series with lengths $N_{1}$ and $N_{2}$ such that $N_{2}=4 N_{1}$. Let RMSE be the measure of accuracy. If the residual $R_{N}$ is random, then we perform simulations to estimate RMSE. We denote the ratio of RMSEs for the window lengths $N_{1}$ and $N_{2}$ by $\Delta=\mathrm{RMSE}_{1} / \mathrm{RMSE}_{2}$. Then, $\Delta=8$ indicates the rate of convergence $1 / N^{1.5}, \Delta=2$ corresponds to the rate of convergence $1 / N^{0.5}$, and $\Delta=1$ means that there is no convergence at all. To estimate $\Delta$, we use $N_{1}=6399$ and $N_{2}=25599$ (we chose odd time series lengths to consider $(N+1) / 2$ as one of window lengths).

We discuss examples with the following three kinds of perturbation of the signal: by a constant, by noise and by a sum of noise and a constant. Also, we consider two types of random noise, white and red. We perform numerical experiments for the time series (3)-(6) with $\sigma=0.1, b=1$, $\alpha=0.5$. In what follows we calculate the frequency and exponential base estimates using the LS-ESPRIT method (the difference with the results of TLS-ESPRIT is small and does not influence the conclusions).

Tables 1-4 include the results on convergence based on 1000 simulations. The column ' $c$ ' corresponds to the time series (3) with constant residuals, the columns 'wn' and 'rn' contain the results for white-noise (the time series (4)) and red-noise (the time series (6)) residuals respectively, and the column 'c+wn' includes estimates of $\Delta$ for the time series (5) with combined perturbation.

It would appear reasonable that the convergence rates for fixed window lengths and for window lengths proportional to $N$ differ. Also, the multiplicity of window lengths to the period of the sine-wave signal (10 in the considered examples) can be important. Therefore, we analyze two sets of window lengths. The first set includes fixed window lengths: the minimal $L=r+1$, where $r=2$ is the rank of sinusoid, $L=20$ is divisible by 10 , and $L=25$ is a common case.
Table 3. The rate of convergence of expon. base estimates: estimated $\Delta$

\begin{tabular}{ccccc}
\hline \hline$L$ & c & c+wn & wn & rn \\
\hline$r+1$ & - & 4.1 & 4.1 & 4.0 \\
20 & - & 4.0 & 4.1 & 3.9 \\
25 & 1.0 & 2.8 & 4.1 & 3.9 \\
\hline$(N+1) / 2-5$ & 16.0 & 8.2 & 8.2 & 8.1 \\
$(N+1) / 2$ & - & 8.2 & 8.2 & 8.1 \\
\hline
\end{tabular}

Table 4. The rate of convergence of frequency estimates: estimated $\Delta$

\begin{tabular}{ccccc}
\hline \hline$L$ & $\mathrm{c}$ & $\mathrm{c}+\mathrm{wn}$ & $\mathrm{wn}$ & $\mathrm{rn}$ \\
\hline$r+1$ & 1.0 & 1.0 & 2.0 & 1.0 \\
20 & - & 2.7 & 2.5 & 1.0 \\
25 & 1.0 & 1.0 & 2.7 & 1.0 \\
\hline$(N+1) / 2-5$ & 16.0 & 8.2 & 8.4 & 8.2 \\
$(N+1) / 2$ & - & 8.2 & 8.4 & 8.2 \\
\hline
\end{tabular}

The second set contains two window lengths close to $N / 2$ : $L=(N+1) / 2$ is divisible by 10 and $L=(N+1) / 2-5$ is a common case. Note that if there is the exact separability (that is possible in the case of deterministic residuals only), then the ratio $\Delta$ cannot be calculated (the sign '-' in the tables). We do not consider the windows length $L>(N+1) / 2$, since these values of $L$ lead to either the same or worse convergence rates in comparison with the window length $N-L+1<(N+1) / 2$.

Let us discuss the results presented in Tables 1-4 for window lengths tending to infinity and for fixed window lengths separately.

Window length $L \sim N / 2$ The simulations provide stable estimates of the convergence rate for the window length $L$ equal to one-half of the time series length (and more generally, for the window lengths that are proportional to $N$ ):

(A) "signal+noise" (the time series (4), (6))

(a) the convergence rate of the projector on the signal subspace is $1 / N^{0.5}$,

(b) the convergence rate of the reconstruction of the whole signal (average error) is $1 / N^{0.5}$,

(c) the convergence rate of the frequency and exponential rate estimates is $1 / N^{1.5}$;

(B) "signal+constant" (the time series (3))

(a) the convergence rate of the projector on the signal subspace is nearly $1 / N$,

(b) the convergence rate of the reconstruction of the whole signal (average error) is nearly $1 / N^{1.5}$,

(c) the convergence rate of the frequency and exponential rate estimates is nearly $1 / N^{2}$.

Theoretical results on the reconstruction errors in a particular case of a noisy constant signal [17], see (7), provide support for a part of the conclusions derived from the simulations. Results on RMSE for the frequency and exponen- 
tial base estimates are confirmed in [2], where the case of a noisy sinusoid is considered. Let us remark that the convergence rate $1 / N^{1.5}$ is not surprising, since the Cramér-Rao lower bound for the variance of the frequency estimates has the same order (see, e.g. [29, 31]). On the other hand, the Cramér-Rao lower bound for the variance of estimates of the sinusoid amplitude has the order $1 / N^{0.5}$, which corresponds to the convergence rate of the reconstruction of the signal. Simulations for the time series (6) confirm that for $L \sim N / 2$ the red-noise residuals provide the same convergence rate as the white-noise residuals.

One can see that the convergence rate in the examples with pure random residuals is much worse than that for the example with deterministic (constant) residuals. As one might expect, the example with combined residuals inherits the worst case. Simulations confirm that in the case of a constant residual mixed with a stochastic component (the time series (5)) and the window length $L$ proportional to $N$, the rate of convergence is the same as for the case $(\mathbf{A})$.

Fixed window length $L=L_{0}$ Let us consider the case of a fixed window length $L$ and $N \rightarrow \infty$. The behavior of the rate of convergence is more complicated than the behavior described above.

Analysis of the reconstruction errors shows the following behavior:

(A) for "signal+noise" (the time series (4), (6)), there is no convergence to the signal, even for the window lengths divisible by the signal (or noise) periods (divisible by $10 \mathrm{in}$ the considered example);

(B) for "signal+constant" (the time series (3)), the convergence holds only if $L$ (or $K=N-L+1$ ) is divisible by the period; in general, there is no convergence.

Consequently, if residuals contain noise, there is no convergence. Thus, in general, small window lengths are not suitable for the problems of signal reconstruction.

Let us now consider the errors of projector and parameter estimates. The difference with the behavior of reconstruction errors consists in the presence of convergence for small $L$ and white-noise residuals. However, the simulations for the time series (6) with red-noise residuals demonstrate the absence of convergence for projection and frequency estimation. In Tables 2 and 4, this absence of convergence corresponds to the values ' 1.0 ' and ' 1.1 ' in the column entitled 'rn'. Convergence of the exponential base estimates still takes place, see Table 3 .

The question is how the deterministic (constant, in our examples) compound of the white-noise residuals influences the convergence for small $L$. Unfortunately, the errors for the projector and parameter estimates converge to 0 for $L=L_{0}$ only if the window length is divisible by the signal periods.

Thus, we can conclude that using small window lengths for frequency estimation is possible only if the residuals are

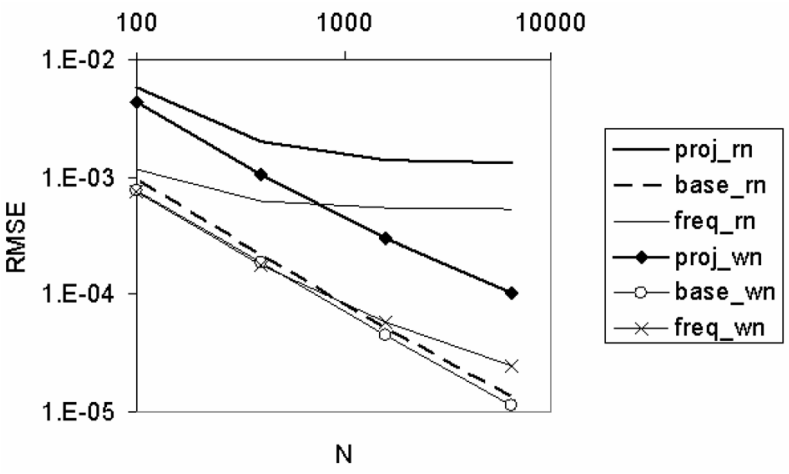

Figure 18. Comparison of RMSE for red-noise and white-noise residuals.

pure white noise, that is, they do not contain deterministic components and are independent. Otherwise, there is no convergence.

Let us compare the estimation error for white and red noise residuals regardless of the absence of convergence in the latter case. Fig. 18 shows the absolute values of the estimation errors for $L=10$. The first symbols of the line titles mean types of the estimated objects: projector ('proj'), exponential base ('base'), or frequency ('freq'); the last symbols designate types of residuals. One can see that the sizes of errors for red and white noise residuals are comparable for the considered time series lengths. This effect is stable enough for different parameters of the time series model. Thus we can perform estimation with good accuracy even in the case of the absence of convergence. This can be explained by the results of [27, formula (2.15)], where the upper bounds for the projector errors are derived. The main term of the upper bound (it is a constant depending on the sinusoid frequency, the parameters of red noise, and the window length) yields the proper order of errors. Moreover, [27, formula (2.9)] provides approximately the same small magnitude $1.3 \cdot 10^{-3}$ as presented in Fig. 18, the line marked 'proj_rn'. Calculations confirm that this magnitude is approximately equal to $K\left(\mathbf{S S}^{\mathrm{T}}\right)^{\dagger} \boldsymbol{\Sigma}\left(\mathbf{I}-\mathbf{U}_{r}^{(s)}\left(\mathbf{U}_{r}^{(s)}\right)^{\mathrm{T}}\right)$, where $\boldsymbol{\Sigma}$ is the autocovariance $L \times L$ matrix of the considered red noise. Note that this term does not converge to 0 as the time series length tends to infinity.

As we have mentioned, the DOA problems use matrices corresponding to the case of fixed $L=L_{0}$, since $L$ is the number of sensors. Therefore, the estimation of frequencies by ESPRIT-like methods can be performed with high accuracy only if the residual is pure white noise (or if $L$ is large enough). In most existing works devoted to the performance of the subspace-based methods for the DOA problems, $L$ is fixed (see, for example, [21] for the root-MUSIC performance or [26] for a wide class of subspace-based algorithms including ESPRIT). Papers [2] and [9] are beyond the scope of DOA and consider the estimation of signal parameters for an arbitrary noisy signal governed by an LRF as $\min (L, K) \rightarrow \infty$. 


\section{CHOICE OF THE WINDOW LENGTH AND SEPARABILITY}

\subsection{Modulated sinusoid}

Note that the ability of SSA-like methods to extract exponentially modulated (damped) sinusoids implies that the SSA method is not simply a spectral method even if we do not apply SSA for trend extraction. Note that both damped and undamped sinusoids have SSA-rank 2 (or rank 1 if cisoids are considered in the complex-valued case). This feature of SSA significantly extends the set of time series that are suitable for the SSA analysis. Most of the classical methods (e.g. Fourier analysis, seasonal decomposition) deal with either constant amplitudes or with amplitudes proportional to the trend (if any) and can be reduced to constant amplitudes by transferring time series to the logarithmic scale. However, time series consisting of several damped sinusoids cannot be reduced to periodic time series or to multiplicative periodicity. Let us consider, for example, a seasonal component containing both yearly and quarterly oscillations and let the amplitude of yearly periodicity increase whereas the amplitude of quarterly oscillations decrease. Then many classical methods fail whereas the SSA-like methods can easily extract such seasonality.

If the behavior of modulations is more complex than the exponential one, then the SSA-like methods can encounter difficulties. These methods can still extract such oscillations, however the question of the proper choice of the window length arises.

Let us formulate the question in a more specific form. Consider the signal in the form of $s_{n}=A(n) \cos (2 \pi n \omega)$, where $A(n)$ is a slowly (in comparison with $\omega$ ) varying function. The question: are there any examples when the choice of the window length close to $N / 2$ is not good.

We consider the time series $f_{n}=s_{n}+r_{n}$ with

$$
s_{n}=\cos (2 \pi n / 19)+\cos (2 \pi n / 21), r_{n}=\varepsilon_{n} .
$$

Here $s_{n}=A(n) \cos (2 \pi n / 20)$ with $A(n)=2 \cos (\pi n(1 / 19-$ $1 / 21)$ ) is a modulated sinusoid of frequency $1 / 20$. The signal has rank 4 and is asymptotically separable from noise, constant residual and others. We have two alternative possibilities. The first possibility is to take $L$ close to $N / 2$ (e.g., between $N / 3$ and $N / 2$ ) and extract the signal by four leading eigentriples. The second alternative is to take a window length $L$ so small that the amplitude of the signal is almost constant within the limits of subseries of length $L$, and then to extract the signal by two leading components. In the latter case, the left singular vectors are close to the undamped sinusoids and the modulation is caught by the right singular vectors.

Numerical simulations (see Fig. 19 for the time series lengths equal to 99, 199, 399, and 999) show that there is no clear choice between the described alternatives. If the time series length is large enough for approximate separability, then the choice $L \sim N / 2$ is better. Otherwise, window

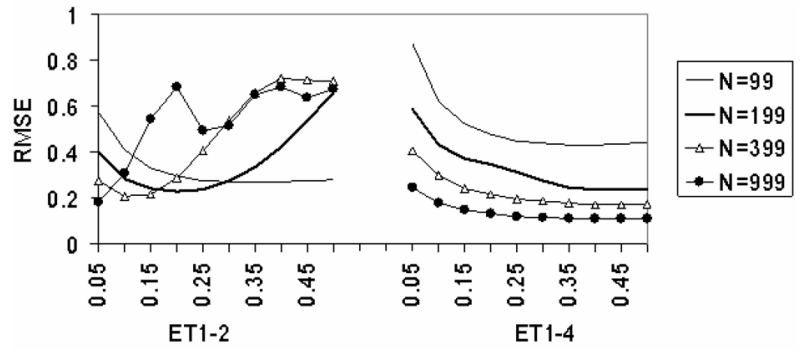

Figure 19. RMSE of reconstruction: dependence on $L / N$ for t.s. (14), ET1-2 and ET1-4.

Table 5. RMSE of reconstruction: different parameter choices for t.s. (14)

\begin{tabular}{cccc}
\hline \hline & $L=40$ & $L=N / 2$ & $L=N / 2$ \\
$\mathrm{~N}$ & $2 \mathrm{ET}$ & $2 \mathrm{ET}$ & $4 \mathrm{ET}$ \\
\hline 99 & $\mathbf{0 . 2 7}$ & $\mathbf{0 . 2 7}$ & 0.45 \\
159 & $\mathbf{0 . 2 3}$ & 0.42 & $\mathbf{0 . 2 4}$ \\
199 & $\mathbf{0 . 2 2}$ & 0.65 & $\mathbf{0 . 2 5}$ \\
399 & $\mathbf{0 . 2 0}$ & 0.70 & $\mathbf{0 . 1 6}$ \\
999 & 0.19 & 0.68 & $\mathbf{0 . 1 1}$ \\
\hline
\end{tabular}

lengths close to a couple of periods provide a better accuracy. The drawback of the latter choice is that usually we do not know the period and therefore cannot guess the proper window length.

Thus, under the conditions of approximate separability (one can check if these conditions are met by means of analysis of the decomposition results, see [15]) the best signal reconstruction uses the window length close to $N / 2$ and the number of the corresponding eigentriples is equal to the signal rank. The advantages of this choice are the better accuracy and the independence of the window length choice from the unknown values of periods. In the considered example, for $L=N / 2$, the reconstruction by four eigentriples performs well starting from $N=160$. However, the choice of $L=N / 2$ is appropriate even for $N<120$ if we produce the reconstruction by two eigentriples. Therefore, there is a limited range of time series lengths (approximately from 120 to 160), where the choice of the window length close to $N / 2$ cannot provide an adequate accuracy of signal reconstruction.

Table 5 contains RMSE for signal reconstruction using two window lengths: $L=40$ (two periods) and $L=N / 2$. The values in bold indicate smaller errors.

The case of complex-form modulation Let us consider the case where the modulated signal is not a signal of finite rank. Let $N=399$ and $f_{n}=s_{n}+r_{n}$, where

$$
s_{n}=A(n) \cos (2 \pi n / 20), \quad r_{n}=\sigma \varepsilon_{n},
$$

where $A(n)=\cos \left(2 \pi n^{2} / 10^{5}\right)$. Figures 20 and 22 show the initial time series with different levels of noise and Figures 21 


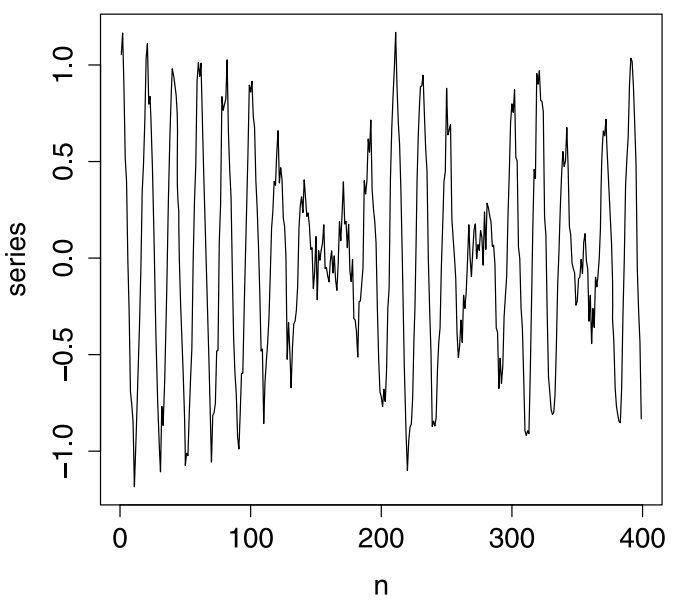

Figure 20. Initial time series: t.s. (15), $\sigma=0.1$.

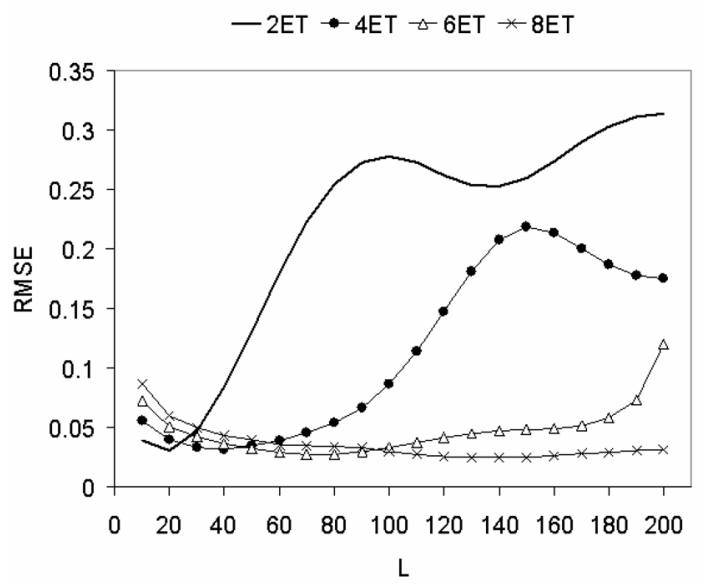

Figure 21. RMSE of reconstruction: t.s. (15), $\sigma=0.1$.

and 23 contain the errors of reconstruction by 2, 4, 6, and 8 leading eigentriples. The choice of the window length for signal reconstruction is not crucial for low levels of noise, since we are able to achieve a good accuracy by choosing eigentriples for reconstruction properly (the larger the window length $L \leq N / 2$, the larger the number of eigentriples chosen for reconstruction).

Fig. 23 shows that for a high level of noise the choice of the window length close to $N / 2$ does not provide the best accuracy. In particular, using $L=200$ with reconstruction by four leading eigentriples is slightly worse than using $L=40$ (two basic periods) and two leading eigentriples. However, if we take, say, $L=80$, then reconstruction by two leading eigentriples is less accurate. Thus, the choice of $L \sim N / 2$ is quite appropriate and can be improved only with the help of additional information (like the value of period) about the analyzed time series.

Note that in this subsection we considered the choice of window lengths for signal reconstruction. Estimation of the

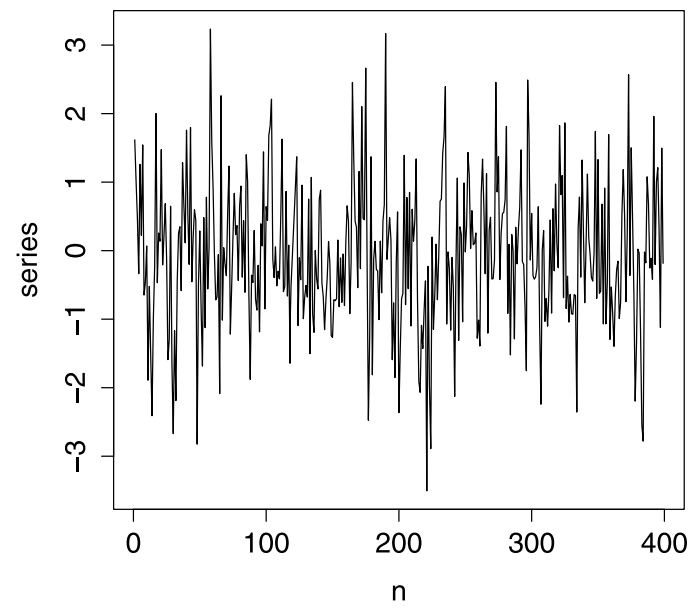

Figure 22. Initial time series: t.s. (15), $\sigma=1$.

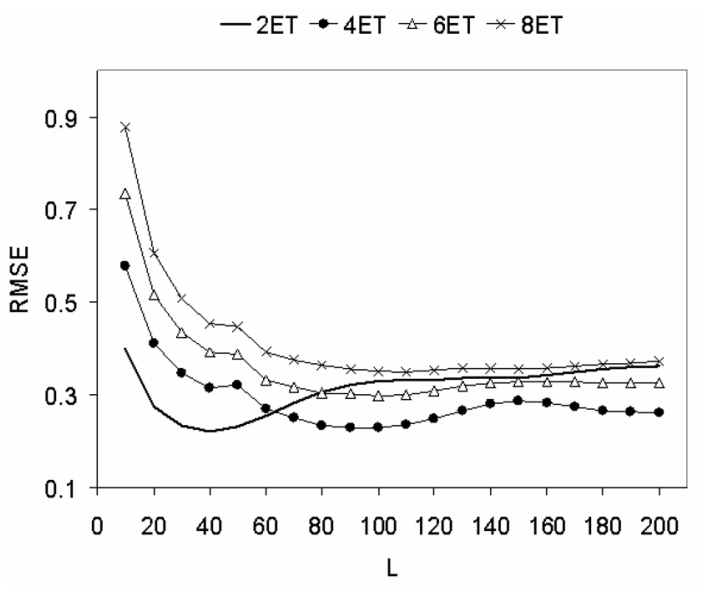

Figure 23. RMSE of reconstruction: t.s. (15), $\sigma=1$. basic frequency (e.g., by ESPRIT) is a separate problem, which requires special statements and approaches to its solution.

\subsection{The problem of mixing}

One of the problems that SSA encounters is a possible lack of strong separability [15] under conditions of weak separability. This problem is caused by equal singular values in SVDs of trajectory matrices of the signal and the residual.

Let us consider some deterministic slowly varying component (i.e., a trend) as a signal. The signal does not necessarily have a finite rank. More likely, it can be approximated by a time series of finite rank $r_{\text {appr }}$. Let $Q$ be the share of $r_{\text {appr }}$ leading squared singular values in the SVD of the trajectory matrix of the trend. Let us denote by $\lambda_{\max }^{\text {resid }}$ the maximal singular value generated by the trajectory matrix of the residual and by $\lambda_{\min }^{\text {trend }}$ the $r_{\text {appr-th singular value of }}$ the trajectory matrix of the trend.

The case of approximate separability corresponds to large 


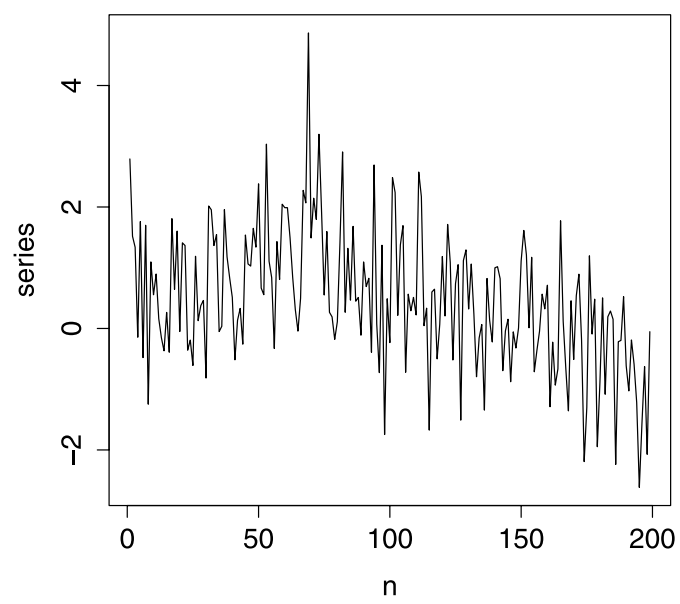

Figure 24. Initial time series: t.s. (16).

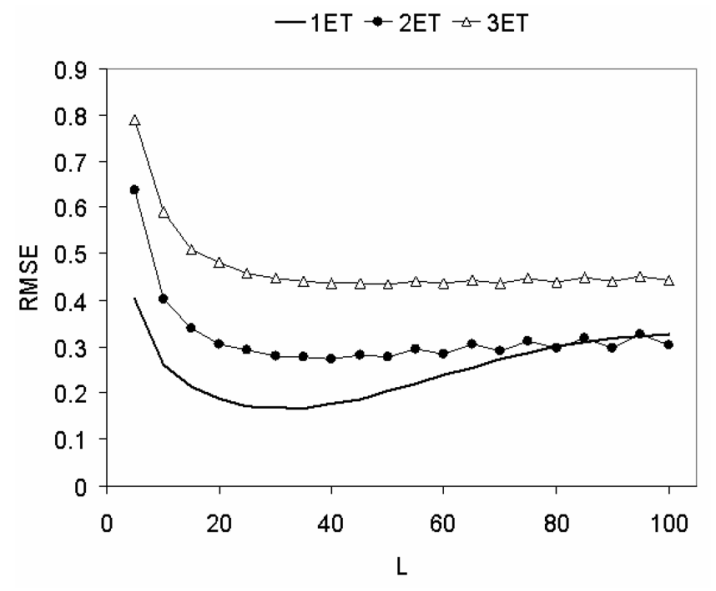

Figure 25. RMSE of reconstruction: t.s. (16).

$Q$ under the condition $\lambda_{\max }^{\text {resid }}<\lambda_{\min }^{\text {trend }}$. If $Q$ is fixed, then, in general, the larger the window length, the larger the rank of the approximating time series and the smaller is $\lambda_{\min }^{\text {trend }}$. This observation can lead to the optimal window lengths considerably smaller than $N / 2$.

We consider the example

$$
s_{n}=\cos \left(2 \pi n^{2} / 10^{5}\right), \quad r_{n}=\varepsilon_{n}+0.5 \cos (2 \pi n / 10),
$$

$N=199$ (Fig. 24). It is easy to check numerically that for $L=100$ we can get $r_{\text {appr }}=2$ with $Q=99.5 \%=92 \%+7.5 \%$ (the singular values are equal to 74 and 21), whereas for $L=30, r_{\text {appr }}=1$ with $Q=98 \%$ (the singular value is 41 ). However, the residual produces the maximal singular value equal approximately to 25 for $L=100$ and to 15 for $L=30$. This means that we have no strong separability for $L=100$ with $Q=99.5 \%$. Thus, we can reach $Q=92.5 \%$ for $L=100$ and $Q=98 \%$ for $L=30$ (certainly, these are just rough measures) to satisfy $\lambda_{\max }^{\text {resid }}<\lambda_{\min }^{\text {trend }}$, that is confirmed by Fig. 25. Note that after extracting the trend with $L_{1}=30$ the periodicity can be extracted from the residual with a window length close to $L_{2}=100$. In [15] this technique is called Sequential SSA.

\section{SSA PROCESSING OF STATIONARY TIME SERIES}

There are special recommendations concerning the choice of parameters for stationary time series. It is traditionally recommended to perform the centering procedure for the stationary time series before processing (i.e., to subtract the average over the time series) and then to use the Toeplitz autocovariance matrix $\widetilde{\mathbf{C}}$ with entries

$$
\widetilde{c}_{i j}=\frac{1}{N-|i-j|} \sum_{m=0}^{N-|i-j|-1} f_{m} f_{m+|i-j|}
$$

instead of $\mathbf{C}=\mathbf{X X}^{\mathrm{T}}$ at the decomposition stage (see [15] for details of the Toeplitz SSA algorithm). Let us remark that using $\mathbf{C}$ to obtain the SVD of the trajectory matrix is sometimes called 'BK' following [6], while using $\widetilde{\mathbf{C}}$ to get the eigenvectors is initiated by the spectral analysis and is called 'VG' following [36]. The use of $\widetilde{\mathbf{C}}$ does not provide us with the SVD of the trajectory matrix and therefore with the SVD optimality.

The papers related to the SSA analysis of climate time series (e.g. [13]) consider the Toeplitz SSA as the main version and state that the Basic and Toeplitz versions only slightly differ. Our investigation shows that Toeplitz SSA provides more stable SSA results (reconstruction, forecast, estimates). However, these results can be inadequate and can have a considerable bias if the time series we analyze is not stationary. It seems that using the Toeplitz version of the SSA algorithm is unsafe if the time series contains a trend or oscillations with increasing or decreasing amplitudes.

Here we can apply a well-known principle: if the method assumes a model, then it gives more precise results when the model is valid; otherwise, the method can produce completely wrong results.

Centering the time series is a less risky procedure than using $\widetilde{\mathbf{C}}$ and can either slightly improve the SSA results or worsen them. Let us note that centering usually increases the rank of the signal making the signal structure more complicated.

Let us recall that besides SSA with centering as preprocessing there are the so called "Single centering SSA" [15], which is appropriate for time series with a constant trend, and also the so called "Double centering SSA" [15], which works well for time series with linear trends.

In the following subsections we demonstrate the examples of application of the centering procedure and the Toeplitz SSA algorithm to non-stationary time series. 


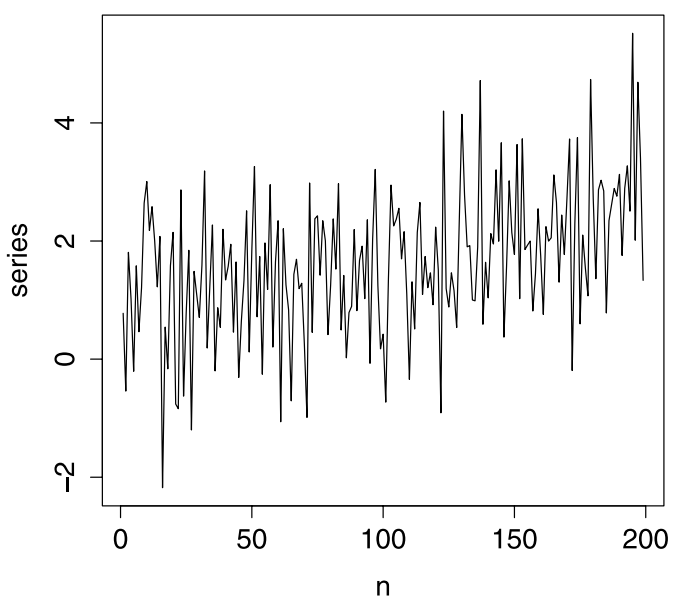

Figure 26. Initial time series: t.s. (17).

\subsection{Centering as preprocessing}

Let $N=199$ and $f_{n}=s_{n}+r_{n}$ with

$$
s_{n}=1.005^{n}, \quad r_{n}=\varepsilon_{n},
$$

see Fig. 26.

The rank of the exponential series is 1 and therefore for its extraction we should choose just one eigentriple. After centering this time series, we obtain a new one $G_{N}=$ $\left(g_{0}, \ldots, g_{N-1}\right)$ with $g_{n}=1.005^{n}-c+\varepsilon_{n}$. Therefore, we should choose two eigentriples to extract $1.005^{n}-c$, i.e., we artificially create a more complex structure of the signal. The results of simulations (see Fig. 27, $L$ changes from 5 to 100 with increment 5) confirm that the thickening of the signal structure ends in the increase of errors.

Practically, the centering procedure can increase the errors of reconstruction even of undamped sinusoids in short time series if the time series length is not divisible by the sine-wave period. The explanation is similar to that for the exponential time series: if the time series length is not divisible by the sine-wave period, then the average over the time series is not equal to 0 . Therefore, after subtracting this average we transform the signal of rank 2 to a signal of rank 3. For long time series the effect of the rank increase is diminished because the time series average is almost zero. However, in such a case centering has no sense.

Remark 8.1 (About filling in the missing data). Let us mention that the method of imputation of missing values introduced in [16] does not imply centering (although centering can be used). In the method from [19] the centering procedure is essential, since the missing values are replaced with zeros at the first iteration. It seems that this peculiarity is caused by the fact that the first applications of SSA have been oriented at stationary time series. Certainly, to generalize the method proposed in [19], we can fill in the missing values using the average over the whole time series or use

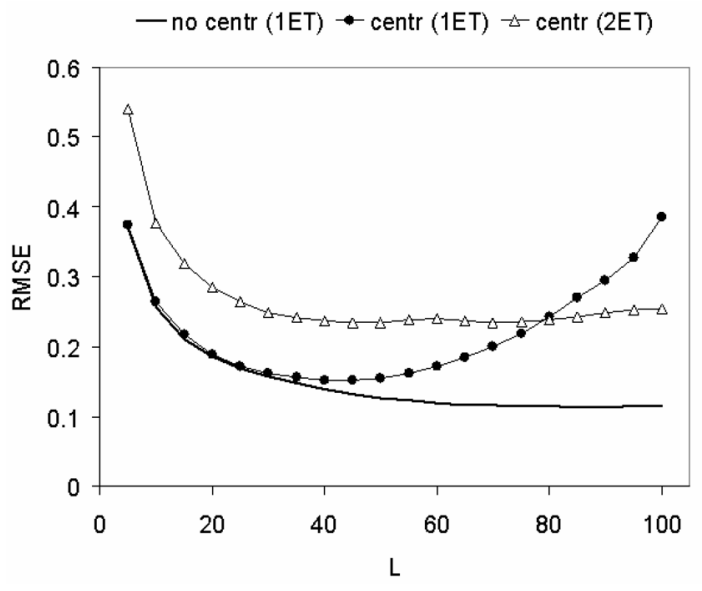

Figure 27. RMSE of reconstruction: t.s. (17), reconstruction by ET1 with no centering and by ET1-2 with the preliminary centering.

an interpolation method based on neighboring non-missing values.

Remark 8.2 (About eigenvalues). Eigenvalues play an important role in the analysis of stationary time series (see, for example, papers devoted to Monte Carlo SSA for detecting a signal in red noise [1]). For stationary time series, the share of eigentriples corresponding to the signal represents the contribution of the corresponding component. However, in the general case of arbitrary time series, there is little point in the eigentriples share, since it depends on absolute values of the times series: in Basic SSA, the distribution of eigenvalues (mostly, leading) depends on the constant compound of the time series while the structure of the time series should not depend on addition or substraction of a constant. In particular, if the leading eigentriple takes $99.9 \%$, this does not mean that it is enough to take only one eigentriple to approximate the time series with high accuracy. Certainly, we can use information on eigenvalues to identify pairs of eigentriples generated by sinusoids (one sinusoid produces two close or equal eigenvalues). However, the same identification can be done by more powerful tools.

\subsection{Toeplitz SSA}

Let us demonstrate the consequences of improper use of Toeplitz SSA. First, application of Toeplitz SSA to nonstationary signals of finite rank $r$ generally increases the number of nonzero eigenvalues from $r$ to the maximal possible value equal to $\min (L, K)$, that is, the structure can be lost. This means that the number of eigentriples required for accurate reconstruction increases. Also, the constructed approximation can have a wrong structure and, for example, lead to a wrong forecast.

We consider two examples of time series of finite rank, $f_{n}=1.005^{n} \cos (2 \pi n / 20)(\operatorname{rank} 2)$ and $f_{n}=1.005^{n}(\operatorname{rank} 1)$. 


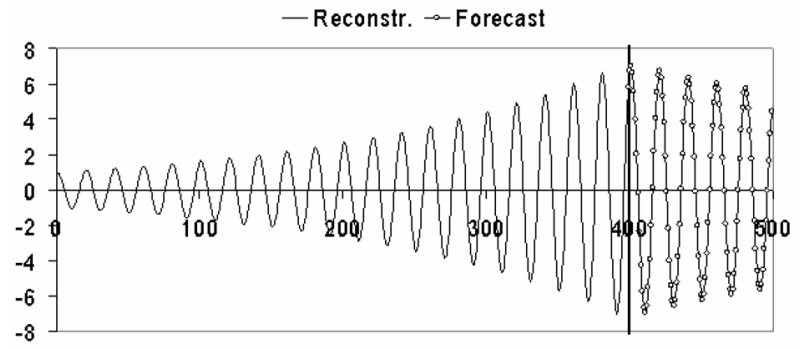

Figure 28. Toeplitz SSA forecast for $f_{n}=1.005^{n} \cos (2 \pi n / 20), N=399, L=200, E T 1-14$.

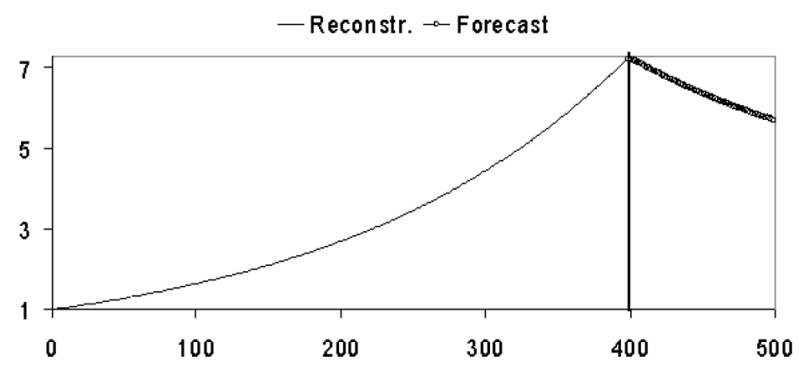

Figure 29. Toeplitz SSA forecast for $f_{n}=1.005^{n}, N=399$, $L=200, E T 1-14$.

To get an accurate approximation of the last time series points, we perform Toeplitz-SSA reconstruction with $L=$ 200 based on ET1-14 (ET1-2 for the first example and ET1 for the second one do not provide good accuracy). Figures 28 and 29 demonstrate that the forecast based on the chosen components is inadequate (moreover, this conclusion does not depend on the chosen window length $L$ and number of components).

One can see that the Toeplitz-SSA forecast is completely wrong while the Basic SSA forecast of finite-rank time series is precise.

\section{SVD-ORIGINS OF SSA AND THE CHOICE OF SSA PARAMETERS}

The key step in SSA is the Singular Value Decomposition (SVD) of the trajectory matrix. The SVD is used for solving different problems, including statistical methods in data analysis. Therefore, the logic of these procedures can also be extended to SSA. Let us briefly describe several origins of SVD-related ideas and views on the SSA-parameter choice generated by them. We do not aim to review the literature on SVD but instead just emphasize the relation between origins and the methodology of SSA.

Principal Component Analysis (PCA) This origin is characterized by different kinds of manipulations with variables and with cases in data, i.e., with rows and columns of the trajectory matrix. In particular, the conventional manipulations are centering and standardization of variables.
This induces different views on eigenvectors and factor vectors (vectors of principal components), where the latter is interpreted as components of the original time series (maybe, just a little shorter than the original time series). In addition, greater attention to eigenvalues' contribution is transferred from PCA to SSA. This attitude to the trajectory matrix is appropriate when we apply Single centering SSA and when the number of rows $(L)$ is fixed and is smaller than the number of columns $(K)$. In the general case, the structure of the trajectory matrix does not depend on the transposition of the trajectory matrix, the interpretation of eigenvalues is not so important and, in particular, Double centering is often more natural than Single one.

Hankel rank-deficient matrices The relation between such matrices and time series governed by linear recurrent formulas has long been known (see e.g. [12]). This technique allows us to analyze noisy time series governed by LRFs. The main application of this idea refers to the signal processing with its approaches to the choice of the method's parameters and consists in the analysis of a noisy sum of damped/undamped cisoids and the estimation of their parameters (mostly, frequencies). However, this approach can be applied to parameter estimation of arbitrary signals governed by LRFs.

Spectral analysis [14] The name of this approach is close to Singular Spectrum Analysis. However, the name 'Spectral analysis' means the analysis of stationary time series and their frequency characteristics. On the other hand, Singular Spectrum is related to the spectrum of linear operators, i.e. singular values of trajectory matrices in the case of SSA. Thus, the analysis of Singular Spectrum does not imply stationarity. As has been stated in [10], the name SSA does not reflect the multifaceted entity of SSA and is traditionally used. Peculiarity of this origin consists in centering the time series before processing and subsequent application of the Toeplitz version of SSA. As was demonstrated in Section 8, if we apply this technique to non-stationary time series, it is likely that we obtain either imprecise or even meaningless results. Within the framework of Spectral analysis approach, special attention is paid to the red noise (autoregression of order 1) and testing the null hypothesis of the absence of a signal in red noise. In some sense, this corresponds to the problem with weak signal and strong noise (in contrast to the previous origin).

Karhunen-Loève expansion [4] This expansion is conventionally used in the theory of stochastic processes and originally assumes zero expectation of elements of the considered stochastic process (or subtracting the known averages). In [4], estimation of the process average is performed by using the moving average. Then the errors from this estimation are included in the centered stochastic process and this allows one to apply the method to processes with trends. Thus, the algorithm appears to be very close to PCA and in fact coincides with Single centering SSA. It seems that 
this origin is used by researchers who are well familiar with the stochastic process techniques and therefore the association with KL expansion helps them to understand the SSA method.

Dynamical systems $[6,11]$ This origin is related to special problems in the theory of dynamical systems with a specific approach to the choice of parameters. However, the contribution of these papers is considerable, since the described algorithm served as an origin of SSA ideas in several applied areas.

\section{CONCLUSION}

In this paper we have considered the SSA-related methods from a unified point of view. The approach to the investigation of these methods was formulated for the 'signal + residual' time series. This enabled us to show the similarity and the specifics of the problems which can be solved by the considered methods.

The accuracy of the methods and the choice of parameters were studied. In the paper we emphasized on the internal mechanism of error origins. The understanding of this internal mechanism together with computer simulations for several typical examples enabled us to formulate the recommendations on the optimal choice of the window length $L$, which is the main parameter in SSA. In particular, the choice of $L$ close to one-half of the time series length was approved as appropriate in most cases. Classes of time series for which this choice should be corrected were indicated.

\section{ACKNOWLEDGMENTS}

The author is thankful to the anonymous referees, the editors and also to Andrey Pepelyshev for their useful comments and suggestions which helped to improve the paper.

\section{Received 5 December 2009}

\section{REFERENCES}

[1] Allen, M. And Smith, L. (1996). Monte Carlo SSA: Detecting irregular oscillations in the presence of colored noise. Journal of Climate 9, 12, 3373-3404.

[2] Badeau, R., Richard, G., And David, B. (2008). Performance of ESPRIT for estimating mixtures of complex exponentials modulated by polynomials. IEEE Transactions on Signal Processing 56, 2, 492-504. MR2445532

[3] Barkhuijsen, H., de Beer, R., and van Ormondt, D. (1987). Improved algorithm for noniterative time-domain model fitting to exponentially damped magnetic resonance signals. J. Magn. Reson. 73, 553-557.

[4] Basilevsky, A. And Hum, D. P. J. (1979). Karhunen-Loéve analysis of historical time series with an application to plantation births in Jamaica. J. Am. Stat. Assoc. 74, 284-290.

[5] Buörck, A. (1996). Numerical Methods for Least Squares Problems. SIAM. MR1386889

[6] Broomhead, D. S. And King, G. P. (1986). Extracting qualitative dynamics from experimental data. Physica D 20, 217-236. MR0859354
[7] Cadzow, J. A. (1988). Signal enhancement: a composite property mapping algorithm. IEEE Transactions on Acoustics, Speech, and Signal Processing 36, 1, 49-62.

[8] DE Groen, P. (1996). An introduction to total least squares. Nieuw Archief voor Wiskunde 14, 237-253. MR1402846

[9] Djermoune, E.-H. And Tomczak, M. (2009). Perturbation analysis of subspace-based methods in estimating a damped complex exponential. IEEE Transactions on Signal Processing 57, 11, 4558-4563.

[10] Elsner, J. B. And Tsonis, A. A. (1996). Singular Spectrum Analysis: A New Tool in Time Series Analysis. Plenum.

[11] Fraedrich, K. (1986). Estimating dimensions of weather and climate attractors. J. Atmos. Sci. 43, 419-432. MR0838629

[12] Gantmacher, F. R. (1959). The Theory of Matrices. Chelsea Publishing Company, New York 68. MR0107649

[13] Ghil, M., Allen, R. M., Dettinger, M. D., Ide, K., Kondrashov, D., Mann, M. E., Robertson, A., Saunders, A., Tian, Y., VAradi, F., And Yiou, P. (2002). Advanced spectral methods for climatic time series. Rev. Geophys. 40, 1, 1-41.

[14] Ghil, M. And VAutard, R. (1991). Interdecadal oscillations and the warming trend in global temperature time series. Nature 350, 324-327.

[15] Golyandina, N., Nekrutkin, V., and Zhigluavsky, A. (2001). Analysis of Time Series Structure: SSA and Related Techniques. Chapman \& Hall/CRC. MR1823012

[16] Golyandina, N. And Osipov, E. (2007). The "Caterpillar"-SSA method for analysis of time series with missing values. J. Stat. Plan. Infer. 137, 8, 2642-2653. MR2326115

[17] Golyandina, N. And Vlassieva, E. (2009). First-order SSAerrors for long time series: model examples of simple noisy signals. In Proceedings of the 6th St.Petersburg Workshop on Simulation Vol.1, June 28-July 4, 2009, St. Petersburg. St. Petersburg State University, 314-319.

[18] Hall, M. J. (1998). Combinatorial theory. Wiley, New York. MR1635901

[19] Kondrashov, D. And Ghil, M. (2006). Spatio-temporal filling of missing points in geophysical data sets. Nonlinear Processes in Geophysics 13, 2, 151-159.

[20] Korobeynikov, A. (2010). Computation- and space-efficient implementation of SSA. Statistics and Its Interface 3, 357-368.

[21] Krim, H., Forster, P., And Proakis, J. G. (1992). Operator approach to performance analysis of root-MUSIC and root-minnorm. IEEE Transactions on Signal Processing 40, 7 (July), 1687-1696.

[22] Kumaresan, R. and Tufts, D. (1982). Estimating the parameters of exponentially damped sinusoids and pole-zero modeling in noise. IEEE Transactions on Acoustics, Speech, and Signal Processing 30, 6, 833-840.

[23] Kumaresan, R. And Tufts, D. W. (1980). Data-adaptive principal component signal processing. In Proc. of IEEE Conference On Decision and Control. Albuquerque, 949-954.

[24] Kumaresan, R. And Tufts, D. W. (1983). Estimating the angles of arrival of multiple plane waves. IEEE Transactions on Aerospace and Electronic Systems AES-19, 1, 134-139.

[25] Kung, S. Y., Arun, K. S., And Rao, D. V. B. (1983). State-space and singular-value decomposition-based approximation methods for the harmonic retrieval problem. J. Opt. Soc. Am. 73, 12, 1799-1811.

[26] Li, F., LiU, H., And Vaccaro, R. J. (1993). Performance analysis for DOA estimation algorithms: unification, simplification, and observations. IEEE Transactions on Aerospace and Electronic Systems 29, 4, 1170-1184.

[27] Nekrutkin, V. (2010). Perturbation expansions of signal subspaces for long signals. Statistics and Its Interface 3, 297-319.

[28] Pakula, L. (1987). Asymptotic zero distribution of orthogonal polynomials in sinusoidal frequency estimation. IEEE Trans. Inf. Theor. 33, 4, 569-576. MR0901683

[29] Rife, D. And Boorstyn, R. (1974). Single tone parameter estimation from discrete-time observations. Information Theory, 
IEEE Transactions on 20, 5, 591-598.

[30] Roy, R. AND KaIlath, T. (1989). ESPRIT: estimation of signal parameters via rotational invariance techniques. IEEE Trans. Acoust. 37, 984-995. MR1058066

[31] Stoica, P., Jakobsson, A., And Li, J. (1997). Cisoid parameter estimation in the colored noise case: asymptotic Cramer-Rao bound, maximum likelihood, and nonlinear least-squares. IEEE Transactions on Signal Processing 45, 8 (Aug.), 2048-2059.

[32] Stoica, P. And Moses, R. (1997). Introduction to Spectral Analysis. Prentice Hall.

[33] Tufts, D. W. and Kumaresan, R. (1982). Estimation of frequencies of multiple sinusoids: Making linear prediction perform like maximum likelihood. Proceedings of the IEEE 70, 9 (Sept.), 975-989.

[34] Usevich, K. (2010). On signal and extraneous roots in Singular Spectrum Analysis. Statistics and Its Interface 3, 281-295.
[35] Van Huffel, S., Chen, H., Decanniere, C., and van Hecke, P. (1994). Algorithm for time-domain NMR data fitting based on total least squares. J. Magn. Reson. Ser. A 110, 228-237.

[36] Vautard, M. And Ghil, M. (1989). Singular spectrum analysis in nonlinear dynamics, with applications to paleoclimatic time series. Physica D 35, 395-424. MR1004204

\section{Nina Golyandina}

Department of Mathematics

St. Petersburg State University

Universitetsky pr. 28,

St. Petersburg, 198504

Russia

E-mail address: nina@gistatgroup.com 